\title{
Collaborative Sparse Regression for Hyperspectral Unmixing
}

\author{
Marian-Daniel Iordache, José M. Bioucas-Dias, Member, IEEE, and Antonio Plaza, Senior Member, IEEE
}

\begin{abstract}
Sparse unmixing has been recently introduced in hyperspectral imaging as a framework to characterize mixed pixels. It assumes that the observed image signatures can be expressed in the form of linear combinations of a number of pure spectral signatures known in advance (e.g., spectra collected on the ground by a field spectroradiometer). Unmixing then amounts to finding the optimal subset of signatures in a (potentially very large) spectral library that can best model each mixed pixel in the scene. In this paper, we present a refinement of the sparse unmixing methodology recently introduced which exploits the usual very low number of endmembers present in real images, out of a very large library. Specifically, we adopt the collaborative (also called "multitask" or "simultaneous") sparse regression framework that improves the unmixing results by solving a joint sparse regression problem, where the sparsity is simultaneously imposed to all pixels in the data set. Our experimental results with both synthetic and real hyperspectral data sets show clearly the advantages obtained using the new joint sparse regression strategy, compared with the pixelwise independent approach.
\end{abstract}

Index Terms-Collaborative sparse regression, hyperspectral imaging, sparse unmixing, spectral libraries.

\section{INTRODUCTION}

$\mathbf{S}$ PECTRAL mixture analysis of remotely sensed hyperspectral images has been a very active research area in recent years, since it faces important challenges [1]-[3]. Linear spectral unmixing [2], [4]-[7] is a standard technique for spectral mixture analysis that infers a set of pure spectral signatures, called endmembers, and the fractions of these endmembers, called abundances, in each pixel of the scene. This model assumes that the spectra collected by the imaging spectrometer can be expressed in the form of a linear combination of endmembers, weighted by their corresponding abundances.

Manuscript received April 30, 2012; revised October 28, 2012; accepted December 30, 2012. This work was supported by the European Community's Marie Curie Research Training Networks Programme under contract MRTN-CT-2006-035927, Hyperspectral Imaging Network (HYPER-I-NET). Funding from the Portuguese Science and Technology Foundation, project PEst-OE/EEI/LA0008/2011, and from the Spanish Ministry of Science and Innovation (CEOS-SPAIN project, reference AYA2011-29334-C02-02) is also gratefully acknowledged.

M.-D. Iordache is with the Flemish Institute for Technological Research (VITO), Centre for Remote Sensing and Earth Observation Processes (TAP), BE-2400 Mol, Belgium (e-mail: diordache@unex.es).

J. M. Bioucas-Dias is with the Telecommunications Institute, Lisbon, 10491, Portugal (e-mail: bioucas@1x.it.pt).

A. Plaza is with the Department of Technology of Computers and Communications, Escuela Politécnica, University of Extremadura, Cáceres, E-10071, Spain (e-mail: aplaza@unex.es).

Color versions of one or more of the figures in this paper are available online at http://ieeexplore.ieee.org.

Digital Object Identifier 10.1109/TGRS.2013.2240001
Given the available spatial resolution of state-of-the-art imaging spectrometers and the presence of the mixture phenomenon at different scales (even at microscopic levels), in some cases the assumption that the remotely sensed data contain one pure observation for each distinct material present in the scene may not be valid [2]. To address this issue, several endmember determination techniques have been developed without assuming the presence of pure signatures in the input data [8]-[12]. This is in contrast with a plethora of algorithms designed under the pure pixel assumption (see [13] and the references therein).

A recently developed approach to tackle the problems related to the unavailability of pure spectral signatures is to model mixed pixel observations as linear combinations of spectra from a library collected on the ground by a field spectro-radiometer. For this purpose, sparse linear regression techniques [14], [15] can be used. Unmixing then amounts to finding the optimal subset of signatures in a (potentially very large) spectral library that can best model each mixed pixel in the scene [16]. In other words, hyperspectral vectors are approximated with a linear combination of a "small" number or regressors (spectral signatures in the library). The regressor weights (fractional abundances) are obtained by minimizing an objective function, often containing a quadratic data term and a sparsity-inducing regularizer, usually the $\ell_{1}$-norm.

Let $\mathbf{A}=\left[\mathbf{a}_{1}, \ldots, \mathbf{a}_{\mathrm{m}}\right]$ denote a spectral library with $m$ spectral signatures, each with $L$ spectral bands. In real applications, the high mutual coherence of the hyperspectral libraries, defined as the largest cosine between any two columns (i.e., $\left.\eta(\mathbf{A}) \equiv \max _{1 \leq k, j \leq m, k \neq j}\left(\left|\mathbf{a}_{k}^{T} \mathbf{a}_{j}\right| /\left\|\mathbf{a}_{k}\right\|_{2}\left\|\mathbf{a}_{j}\right\|_{2}\right)\right)$, imposes limits to the performance of sparse unmixing techniques, namely in what concerns the uniqueness of the solutions [17]. In other words and, as expected, more similar signatures mean more difficult unmixing. Recent works [2] and [16] present a detailed analysis of the strong influence that high mutual coherences of the libraries have on the hyperspectral unmixing solutions. The mutual coherence is also related to the restricted isometric properties (RIP) of the libraries [18] (see also [15], [19] and the variant proposed in [20]), which establish sufficient conditions under which the unmixing solutions can be exactly recovered through linear programming techniques.

In this paper, we exploit the fact that a hyperspectral image always contains a small number of endmembers to remove part of the aforementioned limitations. This means that, if the fractional abundances of the spectral library signatures are collected in a matrix with the number of columns equal to the number of pixels, there should be only a few lines with nonzero entries. In other words, the nonzero abundance lines should appear in a few distinct lines, which implies sparsity 
OBSERVED IMAGE

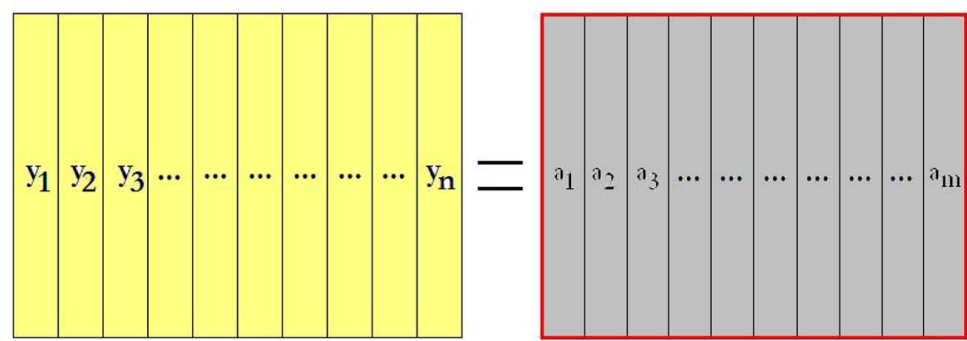

MATRIX OF FRACTIONAL ABUNDANCES

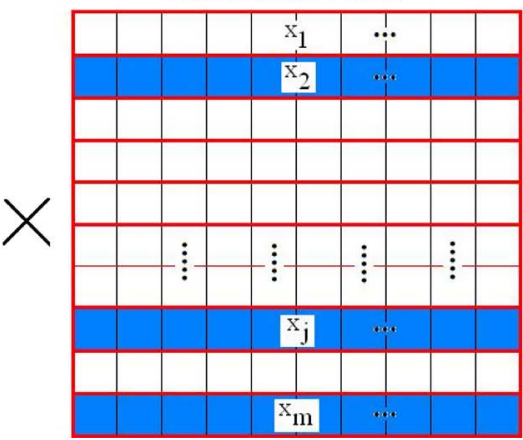

Fig. 1. Graphical illustration of the performance of the proposed collaborative regularizer. Active members of the considered spectral library A are represented in blue color, and non-active members of the considered spectral library $\mathbf{A}$ are represented in white color.

along the pixels of a hyperspectral image in terms of the subpixel information that they convey. At this point, the mutual coherence should have a weaker impact on the unmixing, as the pixels are constrained to share the same active set of endmembers. In fact, the advantages of the collaborative sparse regression approach over the noncollaborative ones have been demonstrated under different approaches (see [21] and the references therein). Here, we exploit the aforementioned observations by adopting a collaborative sparse model strongly related with works [21]-[24] to refine the sparse unmixing methodology. For this purpose, we use a new collaborative sparse regression algorithm based on the ideas described in [25], which constitute a generalization of the sparse unmixing by variable splitting and augmented Lagrangian (SUnSAL) introduced in [26]. Both SUnSAL and the newly developed algorithm, called collaborative SUnSAL (CLSUnSAL) [25], are instances of the methodology introduced in [27].

The remainder of the paper is organized as follows. Section II introduces the proposed collaborative sparse regression framework and summarizes the CLSUnSAL algorithm. Section III shows a quantitative comparison between CLSUnSAL and previous sparse unmixing algorithms, using simulated hyperspectral data. Section IV provides a comparative assessment using a well-known hyperspectral scene collected by the Airborne Visible Infra-Red Imaging Spectrometer (AVIRIS) instrument [28] over the Cuprite mining district in NV. Finally, Section V concludes the paper with some remarks and hints at plausible future research lines.

\section{Collaborative Sparse Regression}

Let $\mathbf{y}$ denote an $L \times 1$ column vector representing an $L$ dimensional pixel vector of a hyperspectral image with $L$ spectral bands. The pixel observation $\mathbf{y}$ can be expressed in terms of a linear combination of spectral signatures in a $L \times m$ spectral library A as follows [16]:

$$
\mathbf{y}=\mathbf{A x}+\mathbf{n}
$$

where $\mathbf{x}$ is an $m \times 1$ vector containing the estimated fractional abundances and $\mathbf{n}$ is an $L \times 1$ vector collecting the errors affecting the measurements at each spectral band. Assuming that the data set contains $n$ pixels organized in the matrix $\mathbf{Y}=\left[\mathbf{y}_{1}, \ldots, \mathbf{y}_{n}\right]$ we may write then

$$
\mathbf{Y}=\mathbf{A X}+\mathbf{N}
$$

where $\mathbf{X}=\left[\mathbf{x}_{1}, \ldots, \mathbf{x}_{n}\right]$ is the abundance fraction matrix and $\mathbf{N}=\left[\mathbf{n}_{1}, \ldots, \mathbf{n}_{n}\right]$ is the noise matrix. The constraints $\mathbf{x} \geq \mathbf{0}$ and $\mathbf{1}^{T} \mathbf{x}=1$ termed, in hyperspectral jargon, abundance nonnegativity constraint (ANC) and abundance sum-to-one constraint (ASC), respectively, are often imposed into the model described in (1) [7].

Let $\|\mathbf{X}\|_{F} \equiv \sqrt{\operatorname{trace}\left\{\mathbf{X} \mathbf{X}^{T}\right\}}$ be the Frobenius norm and $\lambda>0$ denote a regularization parameter. With these definitions in mind, we propose to solve the following optimization problem:

$$
\min _{\mathbf{X}}\|\mathbf{A X}-\mathbf{Y}\|_{F}^{2}+\lambda \sum_{k=1}^{m}\left\|\mathbf{x}^{k}\right\|_{2}
$$

subject to : $\quad \mathbf{X} \geq 0$

where $\mathbf{x}^{k}$ denotes the $k$-th line of $\mathbf{X}$ and $\mathbf{X} \geq 0$ is to be understood componentwise. The convex term $\sum_{k=1}^{m}\left\|\mathbf{x}^{k}\right\|_{2}$ is the so-called $\ell_{2,1}$ mixed norm which promotes sparsity among the lines of $\mathbf{X}$, i.e., it promotes solutions of (2) with small number of nonzero lines of $\mathbf{X}$.

Fig. 1 illustrates the effect of the mixed $\ell_{2,1}$ norm $\sum_{k=1}^{m}\left\|\mathbf{x}^{k}\right\|_{2}$ imposing sparsity among the endmembers simultaneously (collaboratively) for all pixels. CLSUnSAL enforces the presence of the same singletons in the image pixels. Note that a term imposing sparsity for each individual pixel could have been included in the objective function. However, from our experience, the $\ell_{2,1}$ regularizer already imposes sparsity in the solution, making an $\ell_{1}$ term in (2) somehow redundant. On the other hand, the $\ell_{1}$ term slightly improves the unmixing results in certain situations. The great advantage of CLSUnSAL, with the objective function composed by only two terms, is that there is only one regularization parameter used, which strongly alleviates the computational load and the parameter setting process.

Criterion (2) is similar to that of the collaborative (also called "multitask" or "simultaneous") sparse coding problem 
[21]-[24]. The only difference is the introduction of the constraint $\mathbf{X} \geq 0$. Accordingly, we term (2) the constrained collaborative sparse regression (CCSR) problem. Notice that the classical nonnegative constrained least squares (NCLS) solution corresponds to setting $\lambda=0$.

To solve the optimization problem in (2), we use the Collaborative Sparse Unmixing via variable Splitting and Augmented Lagrangian (CLSUnSAL) algorithm [25], which is an ellaboration of the SUnSAL algorithm introduced in [26]. SUnSAL solves the $\ell_{2}+\ell_{1,1}$ norm optimization problem: $\min _{\mathbf{X}}\|\mathbf{A X}-\mathbf{Y}\|_{F}^{2}+\lambda\|\mathbf{X}\|_{1,1}$, subject to $\mathbf{X} \geq 0$, where $\|\mathbf{X}\|_{1,1}=\sum_{j=1}^{n}\left\|\mathbf{x}^{j}\right\|_{1}$ is the $\ell_{1,1}$ norm of $\mathbf{X} \geq 0$ and $\mathbf{x}^{j}$ represents the vector of abundances corresponding to the $j$-th pixel. Here, the first term accounts for the pixel reconstruction error, while the second term imposes sparsity in the solution. The main difference between SUnSAL and CLSUnSAL is that the former employs pixelwise independent regressions, while the latter enforces joint sparsity among all the pixels, which is important in the unmixing due to the fact that the pixels share the same support. CLSUnSAL solves an $\ell_{2}+\ell_{2,1}$ optimization problem in addition to the nonnegativity constraint. CLSUnSAL is an instance of the methodology introduced in [27] for solving $\ell_{2}$ plus a linear combination of convex regularizers, based on the alternative direction method of multipliers (ADMM) [29].

In the literature, many efforts were dedicated to solving problems in which structured variables appear, which is also the case of CLSUnSAL. In [30], the authors study the risk minimization problem for linear supervised learning with regularization by structured sparsity-inducing norms. The risk, in this case, is defined as the sum between a loss function and an $\ell_{2}$ norm regularization term (which, in more general cases, can be replaced by the $\ell_{q}$ norm with $q \geq 1$ ). The $\ell_{2}$ norm applied to overlapping subsets of variables is used to infer allowed nonzero patterns for the solutions. By comparison, CLSUnSAL does not allow overlapping subsets, as all the subsets are considered singletons. In [31], the same structured characteristic of the variables (when the library atoms are organized in groups according to their degree of similarity) is exploited on a per-pixel basis to obtain sparse solutions at both group and individual levels. This approach is known as sparse group lasso and it was adapted for sparse hyperspectral unmixing to minimize the number of groups of materials present in each pixel of an image, treated individually [32]. CLSUnSAL could be also regarded as an instance of the methodology presented in [33], called collaborative hierarchical lasso, which also considers the presence of groups of atoms in the library and imposes sparsity across the pixels, both at group and individual level. The major differences are that CLSUnSAL considers the groups as being singletons while enforcing the ANC. Combined $\ell_{2,1}$ and $\ell_{1,1}$ norm regularizers (i.e., sums of $\ell_{2}$ or $\ell_{1}$ norms, respectively) are used in [34] to deal with multivariate response regression problems in which the data are high-dimensional, but a low number of samples is available. Other works [35], [36] consider tree-organization models of the output to exploit the structured nature of the dictionary or to exploit the group characteristic of the variables. Another library organization was tested in [37], in which the dictionary is organized in two separate blocks, one accounting for texture representation and one dealing with natural scene parts assumed to be piecewise smooth. In the respective work, a total variation (TV) regularizer [38]-[40] is also employed such that the the image fits the piecewise smooth model. The TV regularizer was also used recently in hyperspectral unmixing (see [41]) and, despite the relatively high computational complexity of the model, it brings important improvements in the quality of the final abundance maps. All the aforementioned methods constitute examples of how the a priori information regarding the image (on the one hand) and the library (on the other hand) can be exploited to find sparse reconstructions with high accuracy of the estimated coefficients. In this respect, as mentioned before, CLSUnSAL looks for a structured solution as the matrix of fractional abundances contains only a few nonzero lines. This approach was not used before for hyperspectral unmixing and the theoretical background is supported by the results shown in [21], in which the authors exploit exactly the $\ell_{2,1}$ norm optimization problem for recovering jointly sparse multichannel signals from incomplete measurements.

A valuable theoretical result of [21] is the proof of the superiority that multichannel sparse recovery has over the single channel methods, as the probability of recovery failure decays exponentially in the number of channels. In other words, sparse methods have more chances to succeed when the number of acquisition channels increases, which is extremely important for the sparse unmixing applications, as the number of spectral bands is often in the order of hundreds or even thousands. Herein, we resume the results of Theorem [4.4] in [21], which assumes that the dictionary $\mathbf{A}$ is normalized and composed by i.i.d. Gaussian entries, the observations are generated by a set of atoms whose support is $S \subset\{1,2, \ldots, m\}$ of cardinality $k$ (i.e., there are at most $k$ rows in the solution matrix which are not identically zero) and $\left\|\mathbf{A}_{S}^{+} \mathbf{a}_{l}\right\|_{2} \leq \alpha<1$ holds for all $l \notin S$, where $\mathbf{A}_{S}^{+}$is the pseudoinverse of the matrix $\mathbf{A}_{S}$ containing the atoms from $\mathbf{A}$ corresponding to the indices in $S$. The same Theorem states that, under these assumptions, the solution $\mathbf{X}$ of the linear system of equations $\mathbf{Y}=\mathbf{A X}$ is recovered by solving an $\ell_{2,1}$-norm optimization problem with probability at least $1-m \cdot \exp \left(-(L / 2)\left(\alpha^{-2}-\log \left(\alpha^{-2}\right)-1\right)\right)$. The exponential decay of the error is obvious as $\alpha<1$. Although the conditions from the aforementioned Theorem are not met in common hyperspectral data, in which the dictionary atoms (that is, the pure spectral signatures) are highly correlated leading to high values of $\left\|\mathbf{A}_{S}^{+} \mathbf{a}_{l}\right\|_{2}$, we have systematically observed the same type of behavior in our applications, for which we will give experimental evidence.

We now introduce the details of the CLSUnSAL algorithm. Using the notation $\|\mathbf{X}\|_{2,1}=\sum_{k=1}^{m}\left\|\mathbf{x}^{k}\right\|_{2}$ to denote the $\ell_{2,1}$ norm, the optimization problem (2) can be written in the following equivalent form:

$$
\min _{\mathbf{X}}\|\mathbf{A X}-\mathbf{Y}\|_{F}^{2}+\lambda\|\mathbf{X}\|_{2,1}+\iota_{R+}(\mathbf{X})
$$

where $\iota_{R+}(\mathbf{X})=\sum_{i=1}^{n} \iota_{R+}\left(\mathbf{x}_{i}\right)$ is the indicator function $\left(\mathbf{x}_{i}\right.$ represents the $i$-th column of $\mathbf{X}$ and $\iota_{R+}\left(\mathbf{x}_{i}\right)$ is zero if $\mathbf{x}_{i}$ belongs to the nonnegative orthant and $+\infty$ otherwise). 
The optimization problem (3) has the following (constrained) equivalent formulation:

$$
\begin{aligned}
\min _{\mathbf{U}, \mathbf{V}_{1}, \mathbf{V}_{2}, \mathbf{V}_{3}} \frac{1}{2}\left\|\mathbf{V}_{1}-\mathbf{Y}\right\|_{F}^{2}+\lambda\left\|\mathbf{V}_{2}\right\|_{2,1}+\iota_{R+}\left(\mathbf{V}_{3}\right) \\
\text { subject to } \mathbf{V}_{\mathbf{1}}=\mathbf{A U} \\
\mathbf{V}_{2}=\mathbf{U} \\
\mathbf{V}_{3}=\mathbf{U}
\end{aligned}
$$

which, in compact, form, becomes

$$
\min _{\mathbf{U}, \mathbf{V}} g(\mathbf{V}) \text { subject to } \mathbf{G} \mathbf{U}+\mathbf{B V}=\mathbf{0}
$$

where

$$
\begin{aligned}
\mathbf{V} & \equiv\left(\mathbf{V}_{1}, \mathbf{V}_{2}, \mathbf{V}_{3}\right) \\
g(\mathbf{V}) & \equiv \frac{1}{2}\left\|\mathbf{V}_{1}-\mathbf{Y}\right\|_{F}^{2}+\lambda\left\|\mathbf{V}_{2}\right\|_{2,1}+\iota_{R+}\left(\mathbf{V}_{3}\right) \\
\mathbf{G} & =\left[\begin{array}{c}
\mathbf{A} \\
\mathbf{I} \\
\mathbf{I}
\end{array}\right], \quad \mathbf{B}=\left[\begin{array}{ccc}
-\mathbf{I} & \mathbf{0} & \mathbf{0} \\
\mathbf{0} & -\mathbf{I} & \mathbf{0} \\
\mathbf{0} & \mathbf{0} & -\mathbf{I}
\end{array}\right]
\end{aligned}
$$

Algorithm 1 Alternating direction method of multipliers (ADMM) pseudocode for solving problem (5).

1. Initialization: set $k=0$, choose $\mu>0, \mathbf{U}^{(0)}, \mathbf{V}^{(0)}, \mathbf{D}^{(0)}$

2. repeat:

$$
\begin{aligned}
& \text { 3. } \mathbf{U}^{(k+1)} \leftarrow \arg \min _{\mathbf{U}} \mathcal{L}\left(\mathbf{U}^{(k)}, \mathbf{V}^{(k)}, \mathbf{D}^{(k)}\right) \\
& \text { 4. } \mathbf{V}^{(k+1)} \leftarrow \arg \min _{\mathbf{V}} \mathcal{L}\left(\mathbf{U}^{(k+1)}, \mathbf{V}^{(k)}, \mathbf{D}^{(k)}\right) \\
& \text { 5. } \mathbf{D}^{(k+1)} \leftarrow \mathbf{D}^{(k)}-\mathbf{G} \mathbf{U}^{(k+1)}-\mathbf{B} \mathbf{V}^{(k+1)}
\end{aligned}
$$

6. until some stopping criterion is satisfied.

The ADMM algorithm for the formulation (5) is shown in Algorithm 1, where (see [27], [42])

$$
\mathcal{L}(\mathbf{U}, \mathbf{V}, \mathbf{D}) \equiv g(\mathbf{U}, \mathbf{V})+\frac{\mu}{2}\|\mathbf{G} \mathbf{U}+\mathbf{B V}-\mathbf{D}\|_{F}^{2}
$$

is the augmented Lagrangian for problem (5), $\mu>0$ is a positive constant, and $\mathbf{D} / \mu$ denotes the Lagrange multipliers associated to the constraint $\mathbf{G U}+\mathbf{B V}=\mathbf{0}$. In each iteration, Algorithm 1 sequentially optimizes $\mathcal{L}$ with respect to $\mathbf{U}$ (step 3) and $\mathbf{V}$ (step 4), and then updates the Lagrange multipliers (step 5).

The convergence conditions from [29, Theorem 1] are met: matrix $\mathbf{G}$ is full column rank and function $g$ introduced in (5) is closed, proper, and convex. Under these conditions, the same theorem states that, for any $\mu>0$, if (5) has a solution, say $\mathbf{U}^{*}$, then the sequence $\left\{\mathbf{U}^{(k)}\right\}$ converges to $\mathbf{U}^{*}$. If (5) does not have a solution, then at least one of the sequences $\left\{\mathbf{U}^{(k)}\right\}$ or $\left\{\mathbf{D}^{(k)}\right\}$ diverges. The stopping criterion adopted in the algorithm is $\left\|\mathbf{G} \mathbf{U}^{(k)}+\mathbf{B V}^{(k)}\right\|_{F} \leq \varepsilon$.

In the ADMM scheme, the setting of the parameter $\mu$ has a strong impact over the convergence speed. The evaluation of such a parameter is an active research topic. For example, previous works [43], [44] (see also [45]) use an adaptive scheme (based on the primal and the dual ADMM variables) that performs very well in our case. In this scheme, $\mu$ is updated with the objective of keeping the ratio between the ADMM primal and dual residual norms within a given positive interval, as they both converge to zero.

The details of the optimizations with respect to $\mathbf{U}$ and $\mathbf{V}$ of the ADMM Algorithm 1 (CLSUnSAL) are shown in [25] and we include them in an Appendix, at the end of the paper, for self-contentedness. The optimizations with respect to $\mathbf{V}_{1}$, $\mathbf{V}_{2}$ and $\mathbf{V}_{3}$ are very light. The optimization of $\mathbf{U}$ amounts at solving a linear system of equations of size $m \times m$. The matrix involved in this system of equations is fixed and then can be precomputed involving low complexity as the rank of $\mathbf{A}$ is $\min \{L, m\}$. The optimization with respect to $\mathbf{V}$ is decoupled with respect to $\mathbf{V}_{1}, \mathbf{V}_{2}$ and $\mathbf{V}_{3}$.

Concerning the computational complexity, the most expensive step is the calculus of $\mathbf{U}$, which has the order of complexity $\mathcal{O}\left(n L^{2}\right)$, while the others have complexity $\mathcal{O}(n)$, where $L$ is the number of bands and $n$ is the number of pixels in the image. The overall complexity is, then, $\mathcal{O}\left(n L^{2}\right)$.

\section{Experimental Results With Synthetic Data}

In this section, we give an illustration of the performance of our newly proposed collaborative sparse unmixing approach in a simulated environment. We will compare the results obtained by our proposed method to those obtained with the SUnSAL algorithm [26], and also to the classic NCLS solution. The remainder of the section is organized as follows. Section III-A describes how the simulated data sets have been generated. We consider only scenes affected by noise, because in the noiseless case the true solutions can always be recovered with very high accuracy [2]. Section III-B describes the adopted performance discriminators. Section III-C analyzes the performance of the considered algorithms when the observations are affected by white noise. Finally, Section III-D analyzes the performance of the considered algorithms when the observations are affected by correlated noise.

\section{A. Simulated Datacubes}

The spectral library that we use in our experiments is a dictionary of minerals extracted from the USGS library denoted splib06 ${ }^{1}$ and released in September 2007. It comprises spectral signatures with reflectance values given in 224 spectral bands, distributed uniformly in the interval $0.4-2.5 \mu \mathrm{m}$. Our library, denoted by A, contains $m=240$ members with $L=$ 224 bands. In real applications, it often happens that there are several signatures assigned to a certain endmember, due to the specific conditions of the sample whose spectrum was acquired in the laboratory (e.g., grain size, particle orientation etc.). This is also the case of the considered spectral library $\mathbf{A}$, in which there are 55 materials included, each endmember having a number of variations that ranges between 1 and 17 . The mutual coherence of the library is very close to one. In the library, the signatures are grouped such that the various spectra corresponding to the same endmember are consecutive.

\footnotetext{
${ }^{1}$ Available online: http://speclab.cr.usgs.gov/spectral.lib06
} 


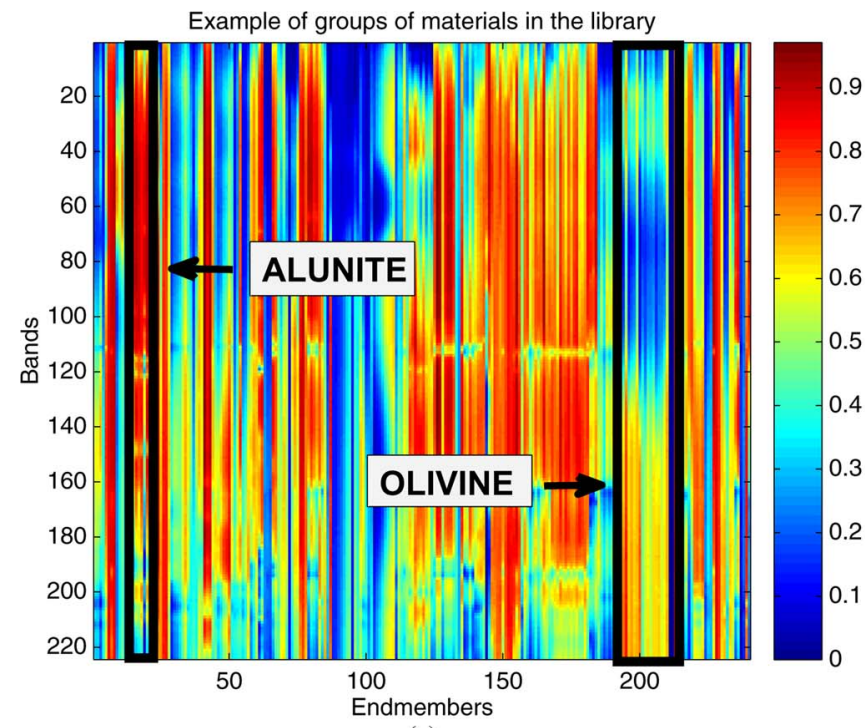

(a)

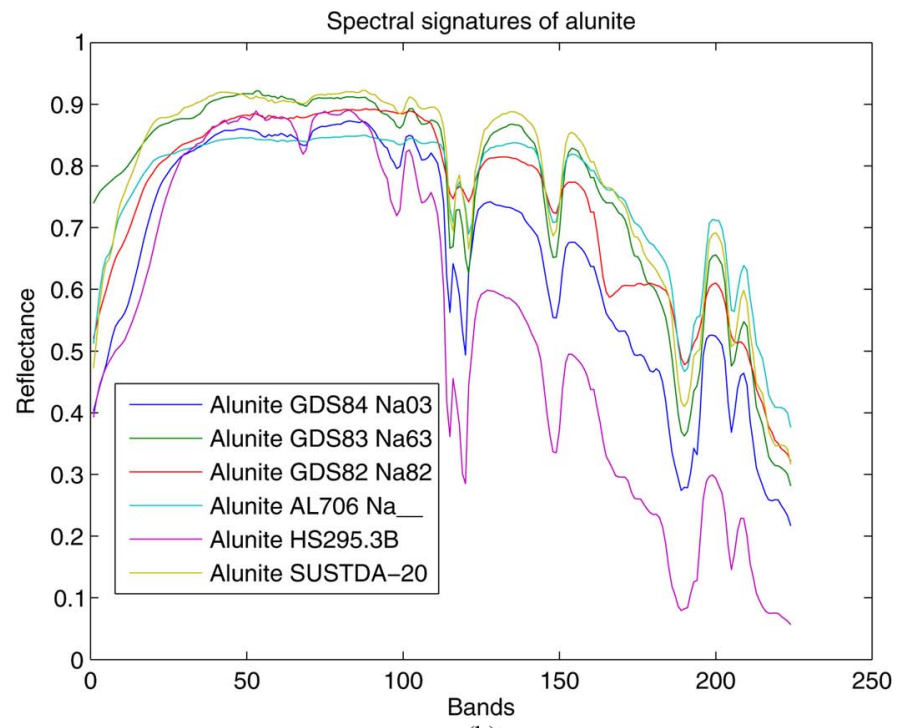

(b)

Fig. 2. (a) Position of two groups of mineral signatures (alunite and olivine) in library A (delimited by black rectangles), and (b) Spectral signatures corresponding to the same material (alunite) included in a group.

As an example, we show in Fig. 2 the position of two groups of mineral signatures (alunite and olivine) within this library [see Fig. 2(a)] and the spectral signatures corresponding to different variations of the same mineral (alunite) collected in one of such groups [see Fig. 2(b)]. We remind that CLSUnSAL does not take into account any group structure. However, the group structure of the library will be used for a more detailed quality assesment of the unmixing results.

Using the library $\mathbf{A}$, we generated various datacubes of 500 pixels, each containing a different number of endmembers: $k_{1}=2$ (generically denoted by DC1), $k_{2}=4$ (DC2) and $k_{3}=$ 6 (DC3). The endmembers were randomly chosen signatures from the library. In each simulated pixel, the fractional abundances of the endmembers follow a Dirichlet distribution [46]. In each group of materials, at most one endmember is used for the generation of the images. The obtained datacubes were then contaminated with both i.i.d. Gaussian noise and correlated noise, having different levels of the signal-to-noise ratio $\operatorname{SNR}(\mathrm{dB})=\mathbb{E}\|\mathbf{A f}\|^{2} / \mathbb{E}\|\mathbf{n}\|^{2}: 20,30$ and $40 \mathrm{~dB}$. The correlated noise was obtained by low-pass filtering i.i.d. Gaussian noise, using a normalized cutoff frequency of $5 \pi / L$. Each noise level corresponds to different sets of endmembers. The rationale behind the experiments with correlated noise is that, as we argued in our previous paper [16], the noise is highly correlated in real hyperspectral applications as it represents mainly modeling noise and the spectra are of low-pass type with respect to the wavelength.

\section{B. Performance Discriminators}

Regarding the performance discriminators adopted in our experiments, the quality of the reconstruction of a spectral mixture was measured using the signal to reconstruction error: $\mathrm{SRE} \equiv E\left[\|\mathbf{x}\|_{2}^{2}\right] / E\left[\|\mathbf{x}-\widehat{\mathbf{x}}\|_{2}^{2}\right]$, expressed in $\mathrm{dB}: \operatorname{SRE}(\mathrm{dB}) \equiv$ $10 \log _{10}$ (SRE). The higher the SRE, the better the quality of the unmixing. We use this measure instead of the classical root-mean-squared error (RMSE) as it gives more information regarding the power of the signal in relation with the power of the error. We also computed a so-called "probability of success", $p_{s}$, which is an estimate of the probability that the relative error power be smaller than a certain threshold. This metric is a widespread one in sparse regression literature, and is formally defined as follows: $p_{s} \equiv P\left(\|\widehat{\mathbf{x}}-x\|^{2} /\|\mathbf{x}\|^{2} \leq\right.$ threshold). For example, if we set threshold $=10$ and get $p_{s}=1$, this means that the total relative error power of the fractional abundances is, with probability one, less than $1 / 10$. This gives an indication about the stability of the estimation that is not inferable directly from the SRE (which is an average). In our case, the estimation result is considered successful when $\|\widehat{\mathbf{x}}-\mathbf{x}\|^{2} /\|\mathbf{x}\|^{2} \leq 3.16(5 \mathrm{~dB})$. This threshold was demonstrated in previous work to provide satisfactory results [16].

In addition, we use the same performance discriminators applied for groups of materials, i.e. by considering that one group represents an endmember and that the sum of the abundances of the group members represent the abundance of the respective endmember. We will define these performance discriminators by $\mathrm{SRE}_{g} \equiv E\left[\left\|\mathbf{x}_{\mathbf{g}}\right\|_{2}^{2}\right] / E\left[\left\|\mathbf{x}_{\mathbf{g}}-\widehat{\mathbf{x}_{\mathbf{g}}}\right\|_{2}^{2}\right]$ measured in dBs: $\operatorname{SRE}_{g}(\mathrm{~dB}) \equiv 10 \log _{10}\left(\mathrm{SRE}_{g}\right)$, and $p_{s, q} \equiv$ $P\left(\left\|\widehat{\mathbf{x}_{\mathrm{g}}}-\mathbf{x}_{\mathbf{g}}\right\|^{2} /\left\|\mathbf{x}_{\mathbf{g}}\right\|^{2} \leq\right.$ threshold $)$, where $\mathbf{x}_{\mathrm{g}}$ and $\widehat{\mathbf{x}_{\mathrm{g}}}$ are vectors whose $j$-th element is the sum of the true and inferred abundances of the members in group $j$, respectively. By using this measure, we will show that, despite the similarity of the signatures, CLSUnSAL is able to better infer the correct endmembers, compared to the methods acting pixelwise.

Moreover, we count the number of nonzero inferred abundances in the two situations: per member (i.e., considering each member of the library as a potential endmember) and per group (i.e., considering each group of materials as a potential endmember). The unmixing problem is solved by NCLS, SUnSAL (which proved to outperform other methods which do not impose sparsity explicitly, see [16]) and CLSUnSAL. For all the algorithms, the parameters were carefully tuned for optimal performance. Next, we describe the results obtained using observations contaminated with white and correlated noise. 
TABLE I

Performance of Different Unmixing Algorithms (Per Member in the Considered Library A) When the Simulated Observations Are AfFected by White Noise

\begin{tabular}{l|c||c|c|c||c|c|c}
\hline \hline & \multicolumn{1}{|c||}{} & \multicolumn{3}{c||}{ SRE $(\mathrm{dB})$} & \multicolumn{3}{c}{$p_{s}$} \\
\hline & SNR & NCLS & SUnSAL & CLSUnSAL & NCLS & SUnSAL & CLSUnSAL \\
\hline DC1 & $20 \mathrm{~dB}$ & 0.69 & 2.37 & 4.85 & 0.16 & 0.18 & 0.44 \\
$\left(k_{1}=2\right)$ & $30 \mathrm{~dB}$ & 7.75 & 8.76 & 11.48 & 0.8 & 0.8 & 1 \\
& $40 \mathrm{~dB}$ & 16.80 & 18.22 & 21.47 & 1 & 1 & 1 \\
\hline $\mathrm{DC} 2$ & $20 \mathrm{~dB}$ & 0.08 & 2.16 & 3.81 & 0.06 & 0.18 & 0.22 \\
$\left(k_{2}=4\right)$ & $30 \mathrm{~dB}$ & 5.2 & 5.22 & 5.93 & 0.56 & 0.56 & 0.58 \\
& $40 \mathrm{~dB}$ & 10.62 & 11.06 & 13.96 & 0.98 & 0.98 & 1 \\
\hline DC3 & $20 \mathrm{~dB}$ & -2.59 & 1.06 & 2.22 & 0.02 & 0.1 & 0.12 \\
$\left(k_{3}=6\right)$ & $30 \mathrm{~dB}$ & 2.87 & 3.26 & 5.31 & 0.36 & 0.36 & 0.56 \\
& $40 \mathrm{~dB}$ & 3.14 & 5.63 & 8.79 & 0.38 & 0.56 & 0.98 \\
\hline \hline
\end{tabular}

TABLE II

Performance of Different Unmixing Algorithms (Per Group in the Considered Library A) When the Simulated Observations Are AfFected by White Noise

\begin{tabular}{l|c||c|c|c||c|c|c}
\hline \hline & \multicolumn{1}{|c||}{} & \multicolumn{3}{c||}{$\mathrm{SRE}_{g}(\mathrm{~dB})$} & \multicolumn{3}{c}{$p_{s, g}$} \\
\hline & SNR & NCLS & SUnSAL & CLSUnSAL & NCLS & SUnSAL & CLSUnSAL \\
\hline DC1 & $20 \mathrm{~dB}$ & 4.97 & 5.46 & 7.58 & 0.5 & 0.5 & 1 \\
$\left(k_{1}=2\right)$ & $30 \mathrm{~dB}$ & 9.34 & 9.5 & 11.36 & 0.86 & 0.86 & 1 \\
& $40 \mathrm{~dB}$ & 18.04 & 20.23 & 23.15 & 1 & 1 & 1 \\
\hline $\mathrm{DC} 2$ & $20 \mathrm{~dB}$ & 2.79 & 3.48 & 4.89 & 0.1 & 0.24 & 0.3 \\
$\left(k_{2}=4\right)$ & $30 \mathrm{~dB}$ & 6.21 & 6.05 & 6.89 & 0.62 & 0.64 & 0.74 \\
& $40 \mathrm{~dB}$ & 15.2 & 15.28 & 17.14 & 1 & 1 & 1 \\
\hline DC3 & $20 \mathrm{~dB}$ & -2.00 & 2.89 & 4.64 & 0.04 & 0.16 & 0.28 \\
$\left(k_{3}=6\right)$ & $30 \mathrm{~dB}$ & 5.9 & 6.84 & 7.95 & 0.66 & 0.74 & 0.82 \\
& $40 \mathrm{~dB}$ & 3.97 & 6.91 & 9.52 & 0.5 & 0.8 & 0.98 \\
\hline \hline
\end{tabular}

\section{Performance in Simulated Data Cubes Contaminated With White Noise}

Table I shows the $\mathrm{SRE}(\mathrm{dB})$ and the $p_{s}$ (per member) obtained after applying NCLS, SUnSAL, and CLSUnSAL to the three simulated datacubes contaminated with white noise. Similarly, Table II shows the same performance indicators, but this time computed per group. From Tables I and II, it can be seen that CLSUnSAL outperforms the other two techniques, both in terms of unmixing accuracy per pixel and per group. As shown by the tables, CLSUnSAL attains the highest SRE(dB) and $p_{s}$ values in all cases. As expected, NCLS exhibits poorer performance than SUnSAL and CLSUnSAL. The accuracy of all algorithms decreases when the cardinality of the solution increases, which is in line with our observations in [16]. This indicates that the sparsity of the solution mitigates the difficulties encountered in unmixing because of the high mutual coherence of the libraries. In all cases, the performance per group is superior to the one corresponding to individual endmembers.

On the other hand, Table III shows the average number of nonzero fractional abundances obtained by each algorithm when calculating the performance metrics reported in Tables I and II, both for individual endmembers in the library and for groups of materials. For simplicity, we declare the fractional abundances larger than 0.001 as "nonzero abundances" to avoid counting negligible values. From Table III it can be observed that NCLS provides solutions containing many nonzero entries (especially when the noise is high) while SUnSAL uses less endmembers and attains better unmixing performance than NCLS. Finally, CLSUnSAL not only provides the most accurate $\operatorname{SRE}(\mathrm{dB})$ and $p_{s}$ values, but also proves to be the algorithm with the sparsest solutions as it uses the lowest number of individual endmembers and groups to explain the data.

\section{Performance in Simulated Data Cubes Contaminated With Correlated Noise}

Table IV shows the $\operatorname{SRE}(\mathrm{dB})$ and the $p_{s}$ (per member) obtained after applying NCLS, SUnSAL and CLSUnSAL to the three simulated datacubes contaminated with correlated noise. Similarly, Table V shows the same performance indicators, but this time computed per group. From Tables IV and V, it can be observed that CLSUnSAL outperforms the other algorithms in terms of $\mathrm{SRE}(\mathrm{dB})$ and $p_{s}$, regardless of whether these metrics are computed per member or per group. This was already the case in the experiments with white noise. As expected, NCLS does not provide optimal performance although it exhibits high values of $p_{s}$ when the noise is low. While SUnSAL is more accurate than NCLS, it is also less accurate than the proposed CLSUnSAL. 
TABLE III

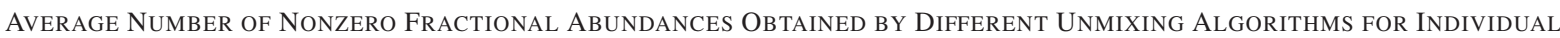
Endmembers and for Groups of Materials When the Simulated ObServations Are AfFected by White Noise

\begin{tabular}{l|c||c|c|c||c|c|c}
\hline \hline & \multicolumn{1}{|c||}{} & \multicolumn{3}{c||}{ Individual endmembers } & \multicolumn{3}{c}{ Groups of materials } \\
\hline & SNR & NCLS & SUnSAL & CLSUnSAL & NCLS & SUnSAL & CLSUnSAL \\
\hline $\mathrm{DC} 1$ & $20 \mathrm{~dB}$ & 60.1 & 42.64 & 12.6 & 19.86 & 16.4 & 10.6 \\
$\left(k_{1}=2\right)$ & $30 \mathrm{~dB}$ & 40.8 & 37.5 & 11.63 & 13.82 & 13.48 & 9.06 \\
& $40 \mathrm{~dB}$ & 30.8 & 37.5 & 9.62 & 11.98 & 11.9 & 8.12 \\
\hline $\mathrm{DC} 2$ & $20 \mathrm{~dB}$ & 75.06 & 40.86 & 11.06 & 21.44 & 15.04 & 10.06 \\
$\left(k_{2}=4\right)$ & $30 \mathrm{~dB}$ & 41.72 & 41.62 & 14.28 & 15.48 & 15.42 & 10.28 \\
& $40 \mathrm{~dB}$ & 34.88 & 34.7 & 9.48 & 13.12 & 13.02 & 8.48 \\
\hline $\mathrm{DC} 3$ & $20 \mathrm{~dB}$ & 92.66 & 39.56 & 11.96 & 23.3 & 14.94 & 9.46 \\
$\left(k_{3}=6\right)$ & $30 \mathrm{~dB}$ & 43.74 & 31.76 & 11.68 & 14.84 & 12.44 & 8.18 \\
& $40 \mathrm{~dB}$ & 40.82 & 26.32 & 10.2 & 14.4 & 10.46 & 6.06 \\
\hline \hline
\end{tabular}

TABLE IV

Performance of Different Unmixing Algorithms (Per Member in the Considered Library A) When the Simulated Observations Are AfFected by Correlated Noise

\begin{tabular}{l|c||c|c|c||c|c|c}
\hline \hline & \multicolumn{1}{|c||}{} & \multicolumn{3}{c||}{ SRE (dB) } & \multicolumn{3}{c}{$p_{s}$} \\
\hline & SNR & NCLS & SUnSAL & CLSUnSAL & NCLS & SUnSAL & CLSUnSAL \\
\hline DC1 & $20 \mathrm{~dB}$ & 2.87 & 5.92 & 9.95 & 0.32 & 0.74 & 0.98 \\
$\left(k_{1}=2\right)$ & $30 \mathrm{~dB}$ & 6.18 & 6.78 & 10.69 & 0.74 & 0.8 & 1 \\
& $40 \mathrm{~dB}$ & 12.27 & 12.51 & 15.9 & 0.98 & 0.98 & 1 \\
\hline $\mathrm{DC} 2$ & $20 \mathrm{~dB}$ & 2.09 & 3.05 & 4.03 & 0.12 & 0.12 & 0.3 \\
$\left(k_{2}=4\right)$ & $30 \mathrm{~dB}$ & 5.11 & 5.92 & 6.13 & 0.44 & 0.46 & 0.58 \\
& $40 \mathrm{~dB}$ & 7.97 & 7.97 & 8.29 & 0.76 & 0.76 & 0.8 \\
\hline $\mathrm{DC} 3$ & $20 \mathrm{~dB}$ & 1.68 & 2.07 & 2.88 & 0.12 & 0.12 & 0.14 \\
$\left(k_{3}=6\right)$ & $30 \mathrm{~dB}$ & 2.5 & 4.37 & 7.28 & 0.3 & 0.38 & 0.76 \\
& $40 \mathrm{~dB}$ & 6.2 & 6.11 & 9.59 & 0.7 & 0.72 & 1 \\
\hline \hline
\end{tabular}

TABLE V

Performance of Different Unmixing Algorithms (Per Group in the Considered Library A) When the Simulated Observations Are AfFected by Correlated Noise

\begin{tabular}{l|c||c|c|c||c|c|c}
\hline \hline & \multicolumn{1}{|c||}{} & \multicolumn{3}{c||}{ SRE $_{g}(\mathrm{~dB})$} & \multicolumn{3}{c}{$p_{s, g}$} \\
\hline & SNR & NCLS & SUnSAL & CLSUnSAL & NCLS & SUnSAL & CLSUnSAL \\
\hline $\mathrm{DC} 1$ & $20 \mathrm{~dB}$ & 3.79 & 6.66 & 10.76 & 0.5 & 0.78 & 0.98 \\
$\left(k_{1}=2\right)$ & $30 \mathrm{~dB}$ & 11.49 & 12.03 & 13.12 & 1 & 1 & 1 \\
& $40 \mathrm{~dB}$ & 12.5 & 12.7 & 16.12 & 1 & 1 & 1 \\
\hline $\mathrm{DC} 2$ & $20 \mathrm{~dB}$ & 3.09 & 3.39 & 5.27 & 0.22 & 0.22 & 0.4 \\
$\left(k_{2}=4\right)$ & $30 \mathrm{~dB}$ & 5.29 & 6.45 & 6.48 & 0.58 & 0.7 & 0.72 \\
& $40 \mathrm{~dB}$ & 8.46 & 8.53 & 10.98 & 1 & 1 & 1 \\
\hline $\mathrm{DC} 3$ & $20 \mathrm{~dB}$ & 1.16 & 2.17 & 2.73 & 0.14 & 0.18 & 0.18 \\
$\left(k_{3}=6\right)$ & $30 \mathrm{~dB}$ & 3 & 5.94 & 8.29 & 0.34 & 0.58 & 0.84 \\
& $40 \mathrm{~dB}$ & 10.02 & 10.12 & 11.45 & 1 & 1 & 1 \\
\hline \hline
\end{tabular}

On the other hand, Table VI shows the average number of nonzero fractional abundances obtained by each algorithm when calculating the performance metrics reported in Tables IV and $\mathrm{V}$, both for individual endmembers in the library and for groups of materials. From Table VI it can be observed that CLSUnSAL uses a much lower number of nonzero abundances than the other two algorithms. In turn, SUnSAL reduces signif- icantly the cardinality of the solution when compared to NCLS but it still needs a larger number of members/groups to explain the observed data as compared to CLSUnSAL.

To illustrate further the advantages of the proposed collaborative framework using computer simulations, Fig. 3 shows a graphical comparison of the performances of the considered unmixing algorithms in a simulated datacube containing 
TABLE VI

Average Number of Nonzero Fractional Abundances Obtained by Different Unmixing Algorithms for Individual Endmembers and For Groups of Materials When the Simulated Observations Are AfFected by Correlated Noise

\begin{tabular}{l|c||c|c|c||c|c|c}
\hline \hline & \multicolumn{1}{|c||}{} & \multicolumn{3}{c||}{ Individual endmembers } & \multicolumn{3}{c}{ Groups of materials } \\
\hline & SNR & NCLS & SUnSAL & CLSUnSAL & NCLS & SUnSAL & CLSUnSAL \\
\hline $\mathrm{DC} 1$ & $20 \mathrm{~dB}$ & 76.48 & 17.54 & 9.5 & 20.9 & 10.5 & 7.04 \\
$\left(k_{1}=2\right)$ & $30 \mathrm{~dB}$ & 17 & 16.54 & 9.3 & 10.44 & 8.98 & 8.22 \\
& $40 \mathrm{~dB}$ & 17.28 & 17.14 & 9.4 & 10.14 & 9.5 & 8.8 \\
\hline $\mathrm{DC} 2$ & $20 \mathrm{~dB}$ & 97.92 & 51.76 & 10.12 & 34.88 & 25.7 & 10.1 \\
$\left(k_{2}=4\right)$ & $30 \mathrm{~dB}$ & 16.38 & 13.4 & 8.48 & 10.5 & 8.4 & 7.88 \\
& $40 \mathrm{~dB}$ & 15.34 & 15.4 & 9.34 & 10.08 & 9.84 & 7.84 \\
\hline $\mathrm{DC} 3$ & $20 \mathrm{~dB}$ & 66.68 & 17.22 & 9.26 & 18.4 & 10.34 & 7.14 \\
$\left(k_{3}=6\right)$ & $30 \mathrm{~dB}$ & 33.72 & 20.82 & 11.38 & 13.76 & 12.6 & 9.64 \\
& $40 \mathrm{~dB}$ & 15.7 & 15.66 & 8.64 & 10.94 & 10.48 & 7.84 \\
\hline \hline
\end{tabular}

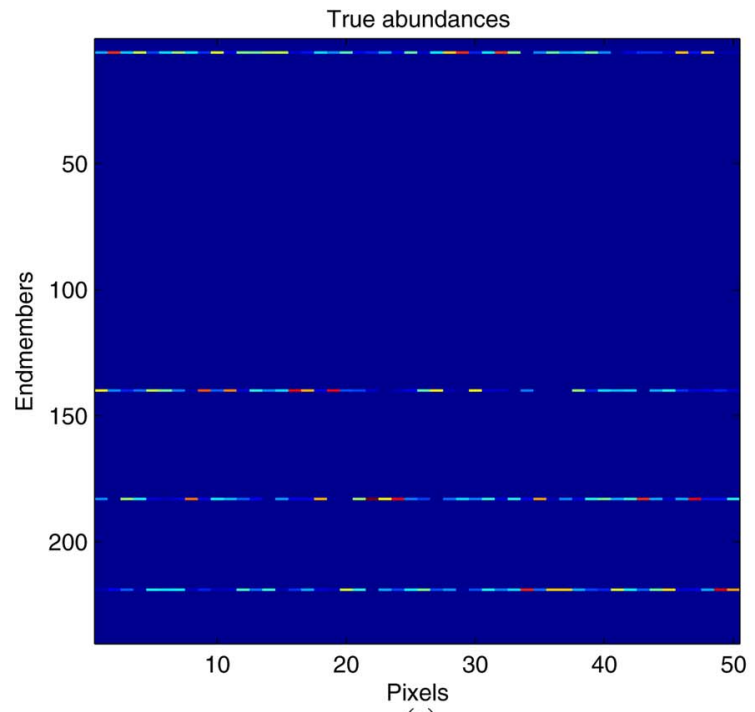

(a)

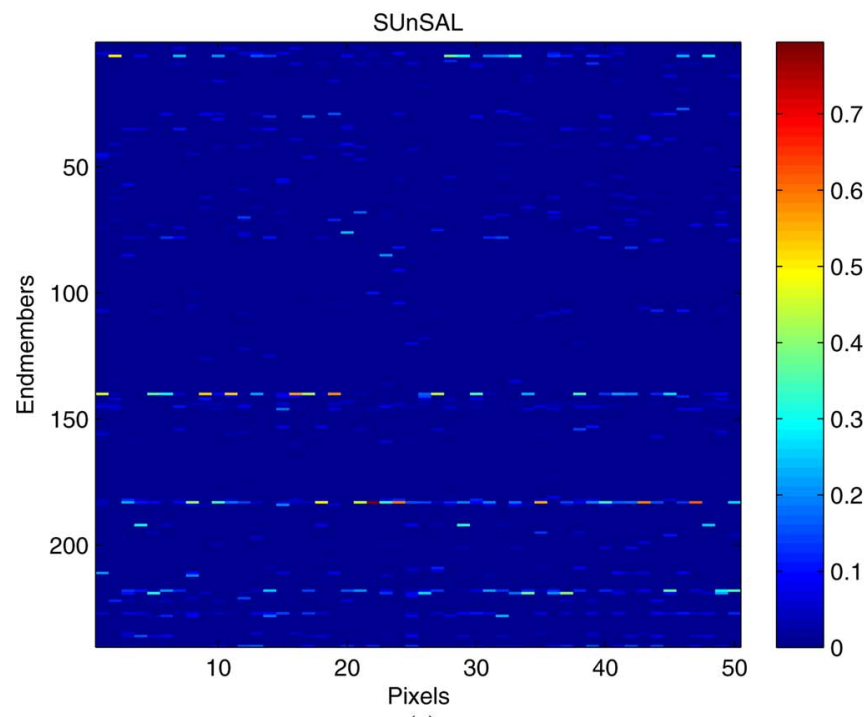

(c)

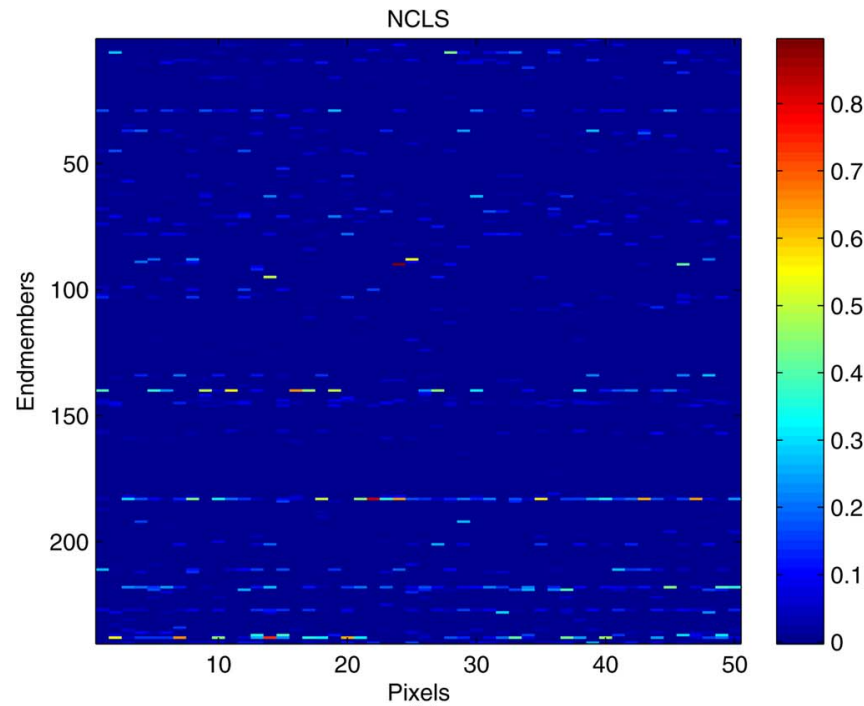

(b)

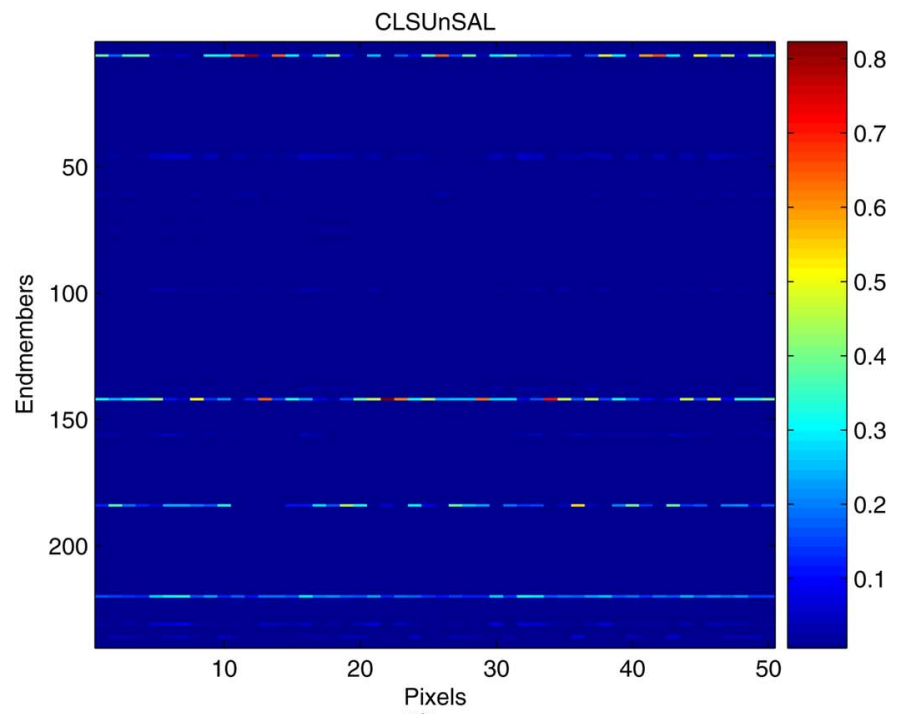

(d)

Fig. 3. (a) Ground-truth abundances in a simulated data set containing 50 pixels and $k=4$ endmembers randomly extracted from library A. The data set is contaminated with correlated noise having SNR of $30 \mathrm{~dB}$. (b) Abundances estimated by NCLS. (c) Abundances estimated by SUnSAL. (d) Abundances estimated by CLSUnSAL.

50 pixels and simulated using $k=4$ endmembers randomly extracted from library $\mathbf{A}$. The datacube was contaminated with correlated noise having SNR of $30 \mathrm{~dB}$. The algorithms were applied after tuning their corresponding parameters to obtain the most accurate solution for each of them. The abundance maps shown in Fig. 3 are in line with our previous observations. 
Note that CLSUnSAL produces abundance maps which are more similar to the ground-truth maps than those produced by the other algorithms. This is because CLSUnSAL constrains the pixels to share the same set of endmembers. Even visually, the superiority of CLSUnSAL with respect to the other algorithms is clearly discernible.

In summary, the experimental results with simulated data cubes reported in this section reveal that CLSUnSAL can improve significantly the cardinalities of the solutions by decreasing the number of nonzero components used to explain the observed data. Further, it is important to emphasize that CLSUnSAL improves significantly the accuracy of the unmixing solutions over those provided by other algorithms such as NCLS or SUnSAL. In all cases, the performances obtained by CLSUnSAL are higher when entire groups of materials are considered as endmembers. This is a consequence of the fact that, due to the noise affecting the simulated scenes, the algorithms are not always able to correctly identify the exact endmembers. Instead, they often identify variations of the respective endmembers present in the spectral library. This is particularly important when one endmember is represented by several variants in the image, indicating the direction toward a possible improvement of the CLSUnSAL algorithm, in which sparsity should be enforced for groups of materials, similar to the work presented in [33]. This will be a research topic for our future work. The results obtained with simulated data sets are very encouraging, but further evaluation with real hyperspectral scenes is highly desirable. This will be accomplished in the following section.

\section{EXPERIMENTAL RESUlts With REAL HYPERSPECTRAL DATA}

The scene used in our real data experiments is the wellknown AVIRIS Cuprite data set, available online in reflectance units. ${ }^{2}$ This scene has been widely used to validate the performance of endmember extraction algorithms. The portion used in experiments corresponds to a $204 \times 151$-pixel subset of the sector labeled as f970619t01p02_r02_sc03.a.rfl in the online data. The scene comprises 224 spectral bands between 0.4 and $2.5 \mu \mathrm{m}$, with nominal spectral resolution of $10 \mathrm{~nm}$. Prior to the analysis, bands $1-2,105-115,150-170$, and 223-224 were removed due to water absorption and low SNR in those bands, leaving a total of 188 spectral bands. The Cuprite site is well understood mineralogically, and has several exposed minerals of interest, all included in the USGS library considered in experiments, denoted splib06 ${ }^{3}$ and released in September 2007. In our experiments, we use spectra obtained from this library as input to the unmixing methods described in Section II. Specifically, the spectral library used in this experiment is the same library A used in our experiments with simulated data. We recall that it contains $m=240$ members corresponding to 55 minerals, with $L=224$ bands. The noisy bands were also removed from A. For illustrative purposes, Fig. 4 shows a mineral map produced in 1995 by USGS, in which the

\footnotetext{
${ }^{2} \mathrm{http} / / /$ aviris.jpl.nasa.gov/html/aviris.freedata.html

${ }^{3}$ http://speclab.cr.usgs.gov/spectral.lib06
}

Tricorder 3.3 software product [47] was used to map different minerals present in the Cuprite mining district. ${ }^{4}$ It should be noted that the Tricorder map is only available for hyperspectral data collected in 1995, while the publicly available AVIRIS Cuprite data was collected in 1997. Therefore, a direct comparison between the 1995 USGS map and the 1997 AVIRIS data is not possible. However, the USGS map serves as a good indicator for qualitative assessment of the fractional abundance maps produced by the unmixing algorithms described in Section II.

Fig. 5 shows a qualitative comparison between the classification maps produced by the USGS Tetracorder algorithm and the fractional abundances inferred by NCLS, SUnSAL and the proposed CLSUnSAL algorithm for four different minerals (alunite, buddingtonite, chalcedony and montmorillonite) which are very prominent in the considered hyperspectral scene. The regularization parameter used for CLSUnSAL in this experiment was empirically set to 0.01 , while the one corresponding to SUnSAL was set to 0.001. As it can be seen in Fig. 5, the unmixing results show a good correlation of the features present in the abundance maps estimated by CLSUnSAL with regard to the classification maps produced by the USGS Tetracorder algorithm. It is also worth noting that the fractional abundances estimated by CLSUnSAL are generally comparable or higher in the regions assigned to the respective materials in comparison to NCLS and SUnSAL. We also emphasize that the average number of endmembers with abundances higher than 0.05 estimated by CLSUnSAL is 5.53 (per pixel), while the average number of groups with total abundances higher than 0.05 is 5.07 . The small difference between these two values leads to the conclusion that CLSUnSAL enforces the sparseness both at the group and individual levels. This means that, inside the selected groups, the algorithm uses a minimum number of members to explain the data. This result is in line with the information provided by the USGS Tetracorder classification map, in which the four selected endmembers are quite dominant in the scene. Overall, the qualitative results reported in this section indicate the improvements that the newly developed CLSUnSAL algorithm can provide by taking advantage of the special characteristics of available spectral libraries and hyperspectral images when conducting the sparse unmixing process.

\section{CONCLusion And Future Work}

In this paper, we have evaluated the performances of a new collaborative sparse regression framework that improves hyperspectral unmixing results by taking advantage of the fact that the number of endmembers in a given hyperspectral scene is generally low and all the observed pixels are generated by the same set of endmembers. These aspects are addressed through a new algorithm called CLSUnSAL which is shown in this work to be able to accurately infer the abundance fractions in both simulated and real environments. The proposed approach reduces the number of endmembers needed to explain the data and provides more robust solutions than those obtained by

${ }^{4}$ http://speclab.cr.usgs.gov/cuprite95.tgif.2.2um_map.gif 




\section{Cuprite, Nevada AVIRIS 1995 Data USGS Clark \& Swayze}

Tricorder 3.3 product

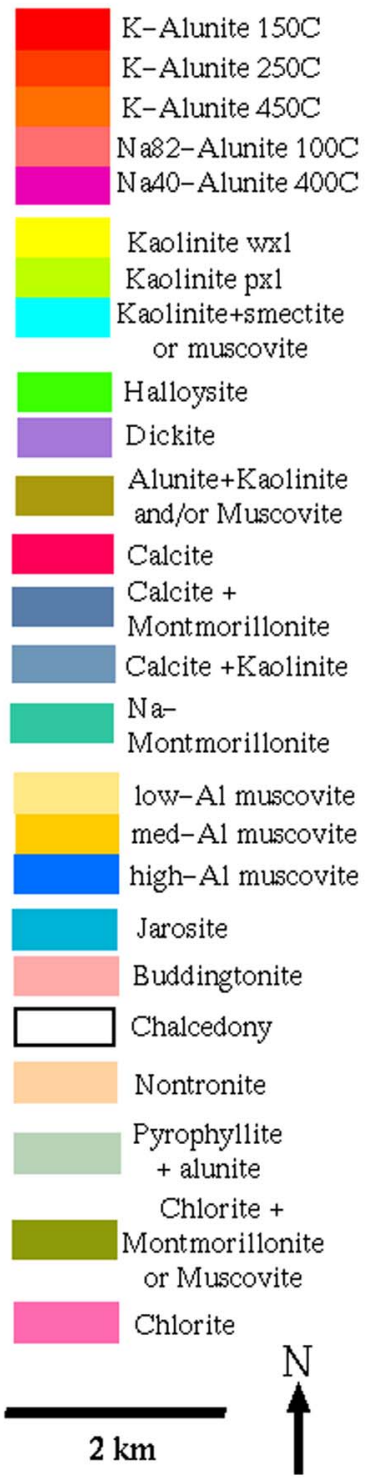

$\mathrm{K}$-Alunite $150 \mathrm{C}$

$\mathrm{K}$-Alunite $250 \mathrm{C}$

$\mathrm{K}$-Alunite $450 \mathrm{C}$

$\mathrm{Na}$ 82-Alunite $100 \mathrm{C}$

unite $400 \mathrm{C}$

Kaolinite px

aolinite+smectite

or muscovite

Halloysite

Dickite

Alunite+Kaolinite

and/or Muscovite

Calcite

Montmorillonite

Calcite + Kaolinite

$\mathrm{Na}-$

high-Al muscovite

Jarosite

yrophyllite

+ alunite

ontmorillonite

or Muscovite

Chlorite

$2 \mathrm{~km}$

Fig. 4. USGS map showing the location of different minerals in the Cuprite mining district in NV. The map is available online at: http://speclab.cr.usgs.gov/cuprite95.tgif.2.2um_map.gif.

other state-of-the-art competitors. Although the experimental results obtained in this paper are very encouraging, further experimentation with additional hyperspectral scenes is needed to fully substantiate our contributions. Also, despite the fact that the proposed algorithm is quite fast as compared to the other tested methods, a possible direction in our future research is the implementation of CLSUnSAL on high performance computing environments to fully exploit its inherently parallel nature to accelerate its computational performance. Other possible improvements of the methodology, including the optimization of objective functions which impose sparsity for groups of materials, will be investigated.

\section{APPENDIX}

In this appendix, we detail the CLSUnSAL algorithm introduced in Section II. We start by expanding the augmented Lagrangian introduced in (6)

$$
\begin{aligned}
\mathcal{L}(\mathbf{U}, & \left.\mathbf{V}_{1}, \mathbf{V}_{2}, \mathbf{V}_{3}, \mathbf{D}_{1}, \mathbf{D}_{2}, \mathbf{D}_{3}\right) \\
= & \frac{1}{2}\left\|\mathbf{V}_{1}-\mathbf{Y}\right\|_{F}^{2}+\lambda\left\|\mathbf{V}_{2}\right\|_{2,1}+\iota_{R+}\left(\mathbf{V}_{3}\right) \\
& +\frac{\mu}{2}\left\|\mathbf{A} \mathbf{U}-\mathbf{V}_{1}-\mathbf{D}_{1}\right\|_{F}^{2}+\frac{\mu}{2}\left\|\mathbf{U}-\mathbf{V}_{2}-\mathbf{D}_{2}\right\|_{F}^{2} \\
& +\frac{\mu}{2}\left\|\mathbf{U}-\mathbf{V}_{3}-\mathbf{D}_{3}\right\|_{F}^{2} .
\end{aligned}
$$




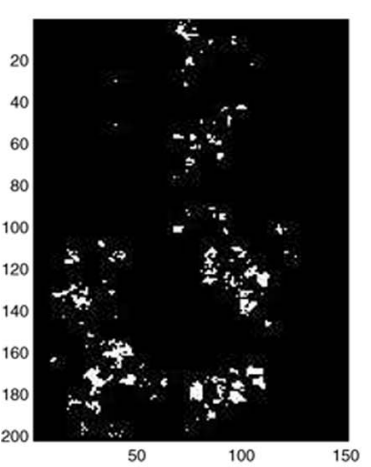

(a)

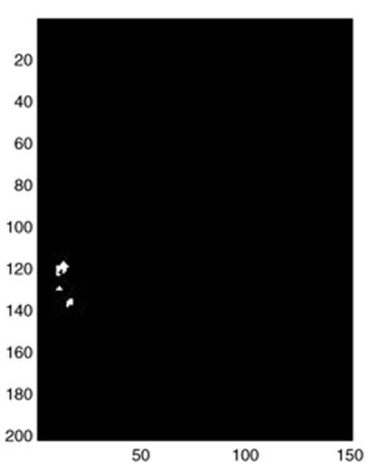

(e)

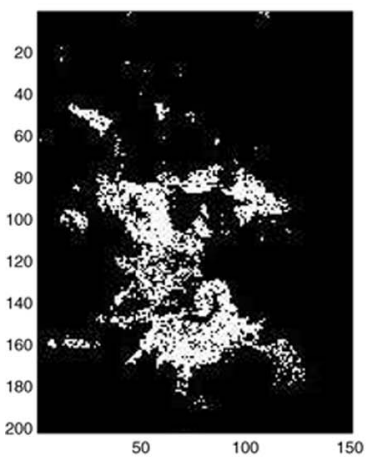

(i)

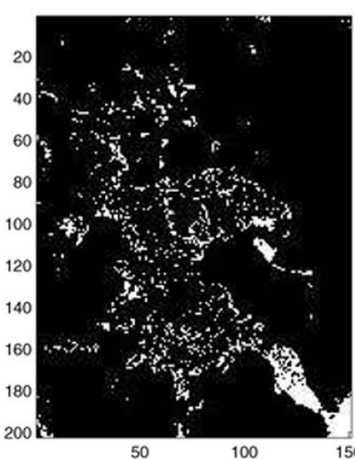

(m)

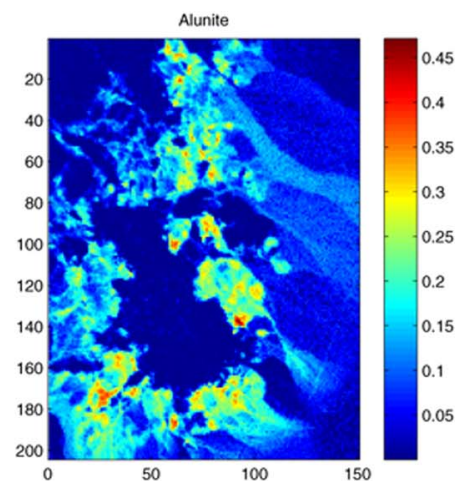

(b)

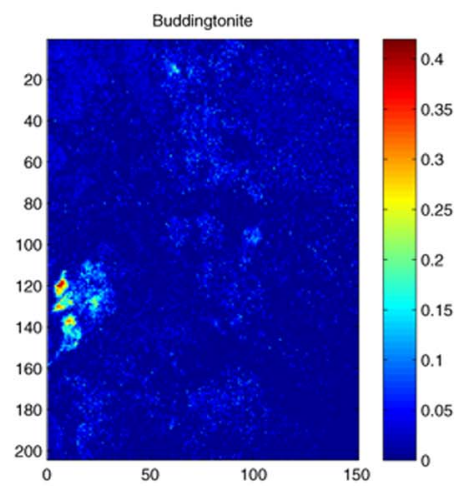

(f)

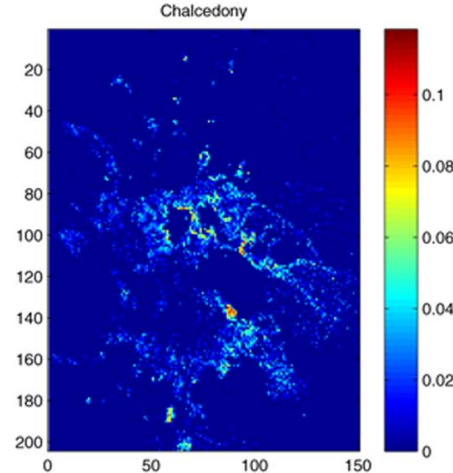

(j)

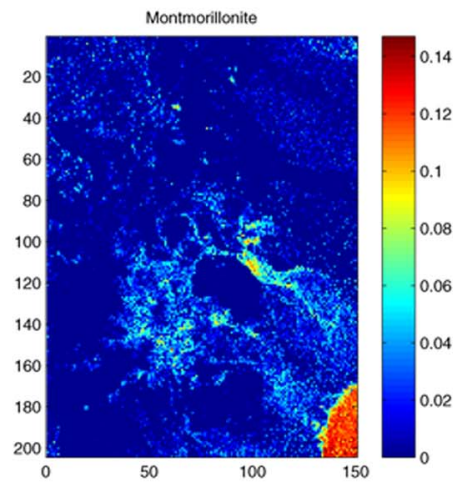

(n)

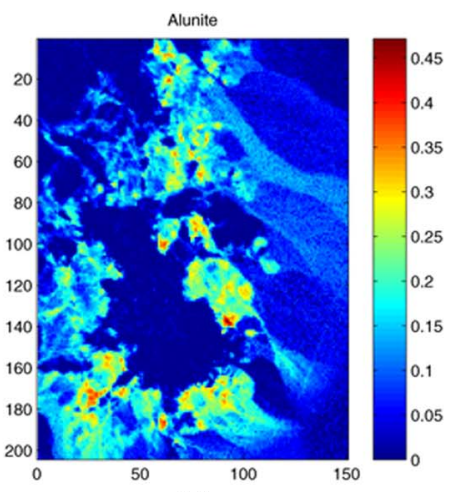

(c)

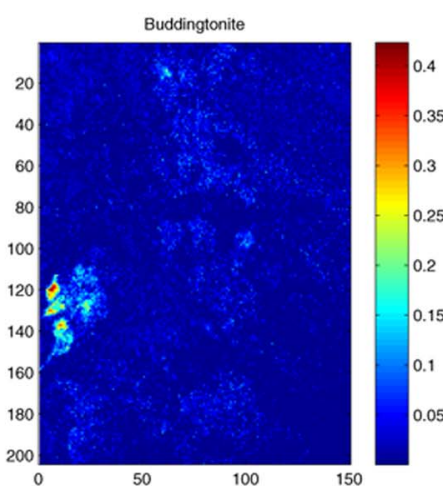

(g)

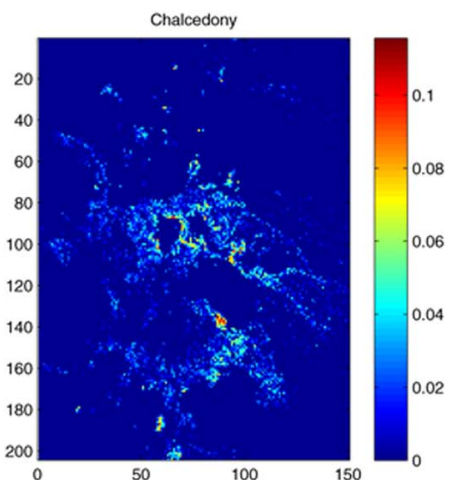

(k)

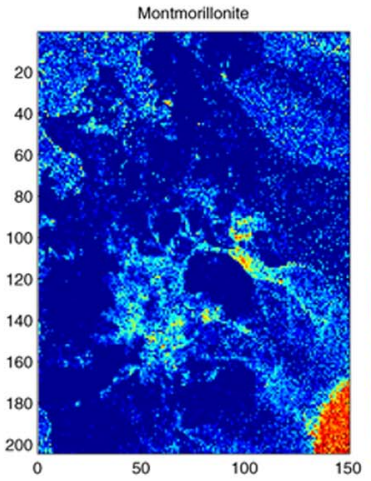

(o)

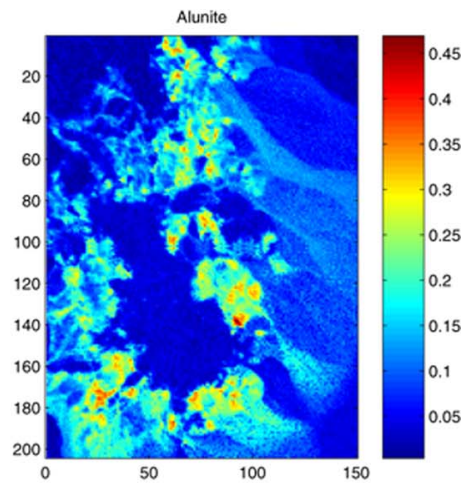

(d)

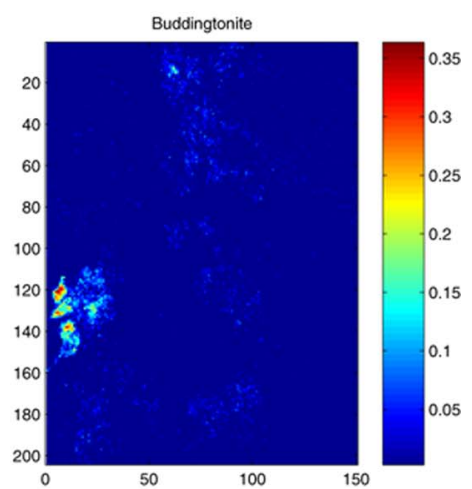

(h)

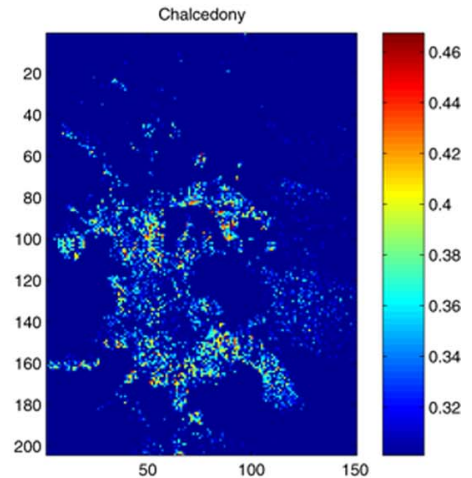

(1)

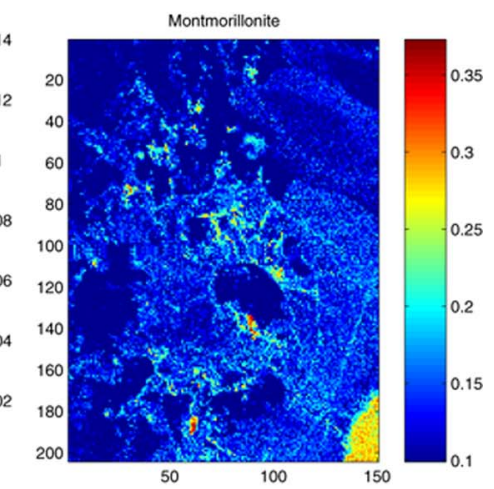

(p)

Fig. 5. Qualitative comparison between the fractional abundance maps estimated by NCLS, SUnSAL, CLSUnSAL and the classification maps produced by USGS Tetracorder for the considered $204 \times 151$-pixel AVIRIS Cuprite scene. (a) USGS Tetracorder classification map for alunite; (b) NCLS abundance map for alunite; (c) SUnSAL abundance map for alunite; (d) CLSUnSAL abundance map for alunite; (e) USGS Tetracorder classification map for buddingtonite; (f) NCLS abundance map for buddingtonite; (g) SUnSAL abundance map for buddingtonite; (h) CLSUnSAL abundance map for buddingtonite; (i) USGS Tetracorder classification map for chalcedony; (j) (f) NCLS abundance map for chalcedony; (j) SUnSAL abundance map for chalcedony; (k) CLSUnSAL abundance map for chalcedony; (1) USGS Tetracorder classification map for montmorillonite; (m) NCLS abundance map for montmorillonite; (n) SUnSAL abundance map for montmorillonite; (o) CLSUnSAL abundance map for montmorillonite. 
The pseudocode of CLSUnSAL is shown in Algorithm 2. It is the expansion of the ADDM algorithm presented in Algorithm 1.

Algorithm 2 Pseudocode of the CLSUnSAL algorithm.

1. Initialization: set $k=0$, choose $\mu \geq 0, \mathbf{U}^{(0)}, \mathbf{V}_{1}^{(0)}, \mathbf{V}_{2}^{(0)}$, $\mathbf{V}_{3}^{(0)}, \mathbf{D}_{1}^{(0)}, \mathbf{D}_{2}^{(0)}, \mathbf{D}_{3}^{(0)}$

2. repeat:

3. $\mathbf{U}^{(k+1)} \leftarrow \arg \min _{\mathbf{U}} \mathcal{L}\left(\mathbf{U}, \mathbf{V}_{1}^{(k)}, \mathbf{V}_{2}^{(k)}, \mathbf{V}_{3}^{(k)}\right.$, $\left.\mathbf{D}_{1}^{(k)}, \mathbf{D}_{2}^{(k)}, \mathbf{D}_{3}^{(k)}\right)$

4. $\mathbf{V}_{1}^{(k+1)} \leftarrow \arg \min _{\mathbf{V}_{1}} \mathcal{L}\left(\mathbf{U}^{(k)}, \mathbf{V}_{1}, \mathbf{V}_{2}^{(k)}, \mathbf{V}_{3}^{(k)}\right)$

5. $\mathbf{V}_{2}^{(k+1)} \leftarrow \arg \min _{\mathbf{V}_{2}} \mathcal{L}\left(\mathbf{U}^{(k)}, \mathbf{V}_{1}^{(k)}, \mathbf{V}_{2}, \mathbf{V}_{3}^{(k)}\right)$

6. $\mathbf{V}_{3}^{(k+1)} \leftarrow \arg \min _{\mathbf{V}_{3}^{(k)}} \mathcal{L}\left(\mathbf{U}^{(k)}, \mathbf{V}_{1}^{(k)}, \mathbf{V}_{2}^{(k)}, \mathbf{V}_{3}\right)$

7. Update Lagrange multipliers:
$\mathbf{D}_{1}^{(k+1)} \leftarrow \mathbf{D}_{1}^{(k)}-\mathbf{A} \mathbf{U}^{(k+1)}+\mathbf{V}_{1}^{(k+1)}$
$\mathbf{D}_{2}^{(k+1)} \leftarrow \mathbf{D}_{2}^{(k)}-\mathbf{U}^{(k+1)}+\mathbf{V}_{2}^{(k+1)}$
$\mathbf{D}_{3}^{(k+1)} \leftarrow \mathbf{D}_{3}^{(k)}-\mathbf{U}^{(k+1)}+\mathbf{V}_{3}^{(k+1)}$

8. Update iteration: $k \leftarrow k+1$

9. until some stopping criterion is satisfied.

The goal of the Step 3 in Algorithm 2 is to determine the value of the variable $\mathbf{U}$ at each iteration. Given that we run an optimization over the variable $\mathbf{U}$, the terms of the objective function (7) which do no contain this variable are not taken into account. The reduced optimization function becomes, then

$$
\begin{aligned}
& \mathbf{U}^{(k+1)} \leftarrow \arg \min _{\mathbf{U}} \frac{\mu}{2}\left\|\mathbf{A} \mathbf{U}-\mathbf{V}_{1}^{(k)}-\mathbf{D}_{1}^{(k)}\right\|_{F}^{2} \\
& \quad+\frac{\mu}{2}\left\|\mathbf{U}-\mathbf{V}_{2}^{(k)}-\mathbf{D}_{2}^{(k)}\right\|_{F}^{2}+\frac{\mu}{2}\left\|\mathbf{U}-\mathbf{V}_{3}^{(k)}-\mathbf{D}_{3}^{(k)}\right\|_{F}^{2} .
\end{aligned}
$$

The solution of (8) is simply

$$
\mathbf{U}^{(k+1)} \leftarrow\left(\mathbf{A}^{T} \mathbf{A}+2 \mathbf{I}\right)^{-1}\left(\mathbf{A}^{T} \xi_{1}+\xi_{2}+\xi_{3}\right)
$$

where $\mathbf{I}$ is the identity matrix, $\mathbf{A}^{T}$ represents the transpose of $\mathbf{A}$ and: $\xi_{1}=\mathbf{V}_{1}^{(k)}+\mathbf{D}_{1}^{(k)}, \xi_{2}=\mathbf{V}_{2}^{(k)}+\mathbf{D}_{2}^{(k)}, \xi_{3}=\mathbf{V}_{3}^{(k)}+$ $\mathbf{D}_{3}^{(k)}$.

Steps 4-6 of CLSUnSAL compute the values of the variables $\mathbf{V}_{1}, \mathbf{V}_{2}, \mathbf{V}_{3}$ at the current iteration. To compute $\mathbf{V}_{1}$, the optimization problem to be solved is

$$
\mathbf{V}_{1}^{(k+1)} \leftarrow \arg \min _{\mathbf{V}_{1}} \frac{1}{2}\left\|\mathbf{V}_{1}-\mathbf{Y}\right\|_{F}^{2}+\frac{\mu}{2}\left\|\mathbf{A} \mathbf{U}^{(k)}-\mathbf{V}_{1}-\mathbf{D}_{1}^{(k)}\right\|_{F}^{2}
$$

whose solution is

$$
\mathbf{V}_{1}^{(k+1)} \leftarrow \frac{1}{1+\mu}\left[\mathbf{Y}+\mu\left(\mathbf{A} \mathbf{U}^{(k)}-\mathbf{D}_{1}^{(k)}\right)\right]
$$

To compute $\mathbf{V}_{2}$, the optimization problem to be solved is

$$
\mathbf{V}_{2}^{(k+1)} \leftarrow \arg \min _{\mathbf{V}_{2}} \lambda\left\|\mathbf{V}_{2}\right\|_{2,1}+\frac{\mu}{2}\left\|\mathbf{U}^{(k)}-\mathbf{V}_{2}-\mathbf{D}_{2}^{(k)}\right\|_{F}^{2}
$$

whose solution is the well-known vect-soft threshold (see, e.g., [48]), applied independently to each row $r$ of the update variable

$$
\mathbf{V}_{2, r}^{(k+1)} \leftarrow \operatorname{vect-soft}\left(\xi_{2, r}, \frac{\lambda}{\mu}\right)
$$

where $\xi_{2}=\mathbf{U}^{(k)}-\mathbf{D}_{2}^{(k)}$ and vect-soft $(\cdot, \tau)$ denotes the rowwise application of the vect-soft-threshold function $\mathbf{b} \mapsto$ $\mathbf{y}\left(\max \left\{\|\mathbf{y}\|_{2}-\tau, 0\right\} / \max \left\{\|\mathbf{y}\|_{2}-\tau, 0\right\}+\tau\right)$.

To compute $\mathbf{V}_{3}$, we solve the optimization problem

$$
\mathbf{V}_{3}^{(k+1)} \leftarrow \arg \min _{\mathbf{V}_{3}} \iota_{R+}\left(\mathbf{V}_{3}\right)+\frac{\mu}{2}\left\|\mathbf{U}^{(k)}-\mathbf{V}_{3}-\mathbf{D}_{3}^{(k)}\right\|_{F}^{2} .
$$

In (14), the role of the $\iota_{R+}$ term is to project the solution onto the nonnegative orthant and the value of $\mathbf{V}_{3}$ is given by

$$
\mathbf{V}_{3}^{(k+1)} \leftarrow \max \left(\mathbf{U}^{(k)}-\mathbf{D}_{3}^{(k)}, 0\right) .
$$

\section{ACKNOWLEDGMENT}

The authors thank Dr. Robert O. Green and Dr. Roger N. Clark for, respectively sharing the AVIRIS Cuprite data and the USGS spectral library with the scientific community. Last but not least, the authors would like to take this opportunity to gratefully thank the Associate Editor and the two Anonymous Reviewers for their comments and suggestions, which greatly helped us to improve the technical quality and presentation of our manuscript.

\section{REFERENCES}

[1] A. Plaza, Q. Du, J. Bioucas-Dias, X. Jia, and F. Kruse, "Foreword to the special issue on spectral unmixing of remotely sensed data," IEEE Trans. Geosci. Remote Sens., vol. 49, no. 11, pp. 4103-4110, Nov. 2011.

[2] J. Bioucas, A. Plaza, N. Dobigeon, M. Parente, Q. Du, P. Gader, and J. Chanussot, "Hyperspectral unmixing overview: Geometrical, statistical, and sparse regression-based approaches," IEEE J. Sel. Topics Appl. Earth Observ. Remote Sens., vol. 5, no. 2, pp. 354-379, Apr. 2012.

[3] M. Parente and A. Plaza, "Survey of geometric and statistical unmixing algorithms for hyperspectral images," in Proc. 2nd WHISPERS, Reykjavik, Iceland, Jun. 14-16, 2010, pp. 1-4.

[4] J. Adams, M. Smith, and P. Johnson, "Spectral mixture modeling: A new analysis of rock and soil types at the Viking Lander 1 site," J. Geophys. Res., vol. 91, no. B8, pp. 8098-8112, Jul. 1986.

[5] A. Plaza, P. Martinez, R. Perez, and J. Plaza, "A quantitative and comparative analysis of endmember extraction algorithms from hyperspectral data," IEEE Trans. Geosci. Remote Sens., vol. 42, no. 3, pp. 650-663, Mar. 2004.

[6] Q. Du, N. Raksuntorn, N. Younan, and R. King, "End-member extraction for hyperspectral image analysis," Appl. Opt., vol. 47, no. 28, pp. 77-84, Oct. 2008.

[7] D. Heinz and C.-I. Chang, "Fully constrained least squares linear spectra mixture analysis method for material quantification in hyperspectral imagery," IEEE Trans. Geosci. Remote Sens., vol. 39, no. 3, pp. 529-545, Mar. 2001.

[8] M. Craig, "Minimum-volume transforms for remotely sensed data," IEEE Trans. Geosci. Remote Sens., vol. 32, no. 3, pp. 542-552, May 1994.

[9] L. Miao and H. Qi, "Endmember extraction from highly mixed data using minimum volume constrained nonnegative matrix factorization," IEEE Trans. Geosci. Remote Sens., vol. 45, no. 3, pp. 765-777, Mar. 2007.

[10] J. Bioucas-Dias, "A variable splitting augmented Lagrangian approach to linear spectral unmixing," in Proc. 1st WHISPERS, Aug. 2009, pp. 1-4.

[11] T.-H. Chan, C.-Y. Chi, Y.-M. Huang, and W.-K. Ma, "A convex analysisbased minimum-volume enclosing simplex algorithm for hyperspectral unmixing," IEEE Trans. Signal Process., vol. 57, no. 11, pp. 4418-4432, Nov. 2009.

[12] J. Bioucas-Dias and J. Nascimento, "Hyperspectral unmixing based on mixtures of Dirichlet components," IEEE Trans. Geosci. Remote Sens., vol. 50, no. 3, pp. 863-878, Mar. 2011. 
[13] J. Bioucas-Dias and A. Plaza, "Hyperspectral unmixing: Geometrical, statistical, and sparse regression-based approaches," in Proc. SPIE, Image Signal Process. Remote Sens. XVI, Toulouse, France, Sep. 20-23, 2010, vol. 7830, pp. 783 00A-1-78300A-15.

[14] B. Natarajan, "Sparse approximate solutions to linear systems," SIAM J. Comput., vol. 24, no. 2, pp. 227-234, Apr. 1995.

[15] E. Candès, J. Romberg, and T. Tao, "Stable signal recovery from incomplete and inaccurate measurements," Commun. Pure Appl. Math., vol. 59, no. 8, pp. 1207-1223, Aug. 2006.

[16] D. Iordache, J. Bioucas-Dias, and A. Plaza, "Sparse unmixing of hyperspectral data," IEEE Trans. Geosci. Remote Sens., vol. 49, no. 6, pp. 2014 2039, Jun. 2011

[17] A. Bruckstein, M. Elad, and M. Zibulevsky, "On the uniqueness of nonnegative sparse solutions to underdetermined systems of equations," IEEE Trans. Inf. Theory, vol. 54, no. 11, pp. 4813-4820, Nov. 2008.

[18] E. Candès and T. Tao, "Decoding by linear programming," IEEE Trans. Inf. Theory, vol. 51, no. 12, pp. 4203-4215, Dec. 2005.

[19] E. Candès, "The restricted isometry property and its implications for compressed sensing," Comp. Rendus Math., vol. 346, no. 9/10, pp. 589592, May 2008

[20] S. Foucart and M. Lai, "Sparsest solutions of underdetermined linear systems via lq-minimization for $0<q \leq 1$," Appl. Comput. Harmonic Anal., vol. 26, no. 3, pp. 395-407, May 2009.

[21] Y. Eldar and H. Rauhut, "Average case analysis of multichannel sparse recovery using convex relaxation," IEEE Trans. Inf. Theory, vol. 56, no. 1, pp. 505-519, Jan. 2010.

[22] J. Tropp, "Algorithms for simultaneous sparse approximation. Part ii: Convex relaxation," Signal Process., vol. 86, no. 3, pp. 589-602, Mar. 2006.

[23] M. Mishali and Y. Eldar, "Reduce and boost: Recovering arbitrary sets of jointly sparse vectors," IEEE Trans. Signal Process., vol. 56, no. 10, pp. 4692-4702, Oct. 2008.

[24] B. Turlach, W. Venables, and S. Wright, "Simultaneous variable selection," Technometrics, vol. 47, no. 3, pp. 349-363, Aug. 2004.

[25] J. Bioucas-Dias and M. Figueiredo, "An Alternating Direction Algorithm for Collaborative Hierarchical Sparse Regularization," IST, Lisbon, Portugal, Tech. Rep., Sep. 2011.

[26] J. Bioucas-Dias and M. Figueiredo, "Alternating direction algorithms for constrained sparse regression: Application to hyperspectral unmixing," in Proc. 2nd WHISPERS, 2010, pp. 1-4.

[27] M. Afonso, J. Bioucas-Dias, and M. Figueiredo, "An augmented Lagrangian approach to the constrained optimization formulation of imaging inverse problems," IEEE Trans. Image Process., vol. 20, no. 3, pp. 681-695, Mar. 2011.

[28] R. Green, M. Eastwood, C. Sarture, T. Chrien, M. Aronsson, B. Chippendale, J. Faust, B. Pavri, C. Chovit, M. Solis, M. R. Olah, and O. Williams, "Imaging spectroscopy and the airborne Visible/Infrared Imaging Spectrometer (AVIRIS)," Remote Sens. Environ., vol. 65, no. 3, pp. 227-248, Sep. 1998

[29] J. Eckstein and D. Bertsekas, "On the DouglasRachford splitting method and the proximal point algorithm for maximal monotone operators," Math. Programm., vol. 55, no. 3, pp. 293-318, Jun. 1992.

[30] R. Jenatton, J. Audibert, and F. Bach, "Structured variable selection with sparsity-inducing norms," arXiv, 2009, 0904.3523v1.

[31] J. Friedman, T. Hastie, and R. Tibshirani, "A note on the group Lasso and a sparse group Lasso (Preprint)," arXiv, 2010, 1001.0736v1 [math.ST].

[32] M.-D. Iordache, J. Bioucas-Dias, and A. Plaza, "Hyperspectral unmixing with Sparse Group Lasso," in Proc. IEEE IGARSS, Vancouver, Canada, 2011, pp. 3586-3589.

[33] P. Sprechmann, I. Ramirez, G. Sapiro, and Y. Eldar, "C-hilasso: A collaborative hierarchical sparse modeling framework," IEEE Trans. Signal Process., vol. 59, no. 9, pp. 4183-4198, Sep. 2011.

[34] J. Peng, J. Zhu, A. Bergamaschi, W. Han, D. Noh, J. Pollack, and P. Wang, "Regularized multivariate regression for identifying master predictors with application to integrative genomics study of breast cancer," Ann. Appl. Stat., vol. 4, no. 1, pp. 53-77, Mar. 2010.

[35] S. Kim and E. P. Xing, "Tree-guided group lasso for multi-task regression with structured sparsity," in Proc. ICML, 2010, pp. 543-550.

[36] J. R, J. Mairal, G. Obozinski, and F. Bach, "Proximal methods for sparse hierarchical dictionary learning," in Proc. ICML, 2010, pp. 487-494.

[37] J.-L. Starck, M. Elad, and D. Donoho, "Image decomposition via the combination of sparse representations and a variational approach," IEEE Trans. Image Process., vol. 14, no. 10, pp. 1570-1582, Oct. 2005.

[38] L. Rudin, S. Osher, and E. Fatemi, "Nonlinear total variation based noise removal algorithms," Phys. D, vol. 60, no. 1-4, pp. 259-268, Nov. 1992.

[39] Z. Guo, T. Wittman, and S. Osher, "L1 unmixing and its application to hyperspectral image enhancement," in Proc. SPIE Conf. Algorithms
Technol. Multispectr., Hyperspectr., Ultraspectr. Imagery XV, Orlando, FL, USA, 2009, vol. 7334, pp. 73 341-73 449

[40] A. Chambolle, "An algorithm for total variation minimization and applications," J. Math. Imaging Vis., vol. 20, no. 1/2, pp. 89-97, Jan. 2004.

[41] D. Iordache, J. Bioucas-Dias, and A. Plaza, "Total variation spatial regularization for sparse hyperspectral unmixing," IEEE Trans. Geosci. Remote Sens., vol. 50, no. 11, pp. 4484-4502, Nov. 2011

[42] E. Esser, "Applications of Lagrangian-Based Alternating Direction Methods and Connections to Split-Bregman," Univ. California, Berkeley, CA, USA, Tech. Rep. 09-31, 2009.

[43] B. He and S. Wang, "Alternating direction method with self-adaptive penalty parameters for monotone variational inequalities," J. Optim. The ory Appl., vol. 106, no. 2, pp. 337-356, Aug. 2000.

[44] S. L. Wang and L. Z. Liao, "Decomposition method with a variable parameter for a class of monotone variational inequality problems," J. Optim. Theory Appl., vol. 102, no. 2, pp. 415-429, May 2001.

[45] S. Boyd, N. Parikh, E. Chu, B. Peleato, and J. Eckstein, "Distributed optimization and statistical learning via the alternating direction method of multipliers," Found. Trends Mach. Learn., vol. 3, no. 1, pp. 1-122, Jan. 2011.

[46] J. Nascimento and J. Bioucas-Dias, "Vertex component analysis: A fast algorithm to unmix hyperspectral data," IEEE Trans. Geosci. Remote Sens., vol. 43, no. 4, pp. 898-910, Apr. 2005.

[47] R. Clark, G. Swayze, K. Livo, R. Kokaly, S. Sutley, J. Dalton, R. McDougal, and C. Gent, "Imaging spectroscopy: Earth and planetary remote sensing with the USGS Tetracorder and expert systems," J. Geophys. Res., vol. 108, no. E12, pp. 5131-5135, Dec. 2003.

[48] S. Wright, R. Nowak, and M. Figueiredo, "Sparse reconstruction by separable approximation," IEEE Trans. Signal Process., vol. 57, no. 7, pp. 2479-2493, Jul. 2009.

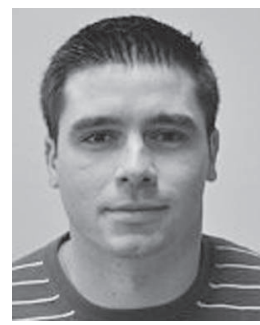

Marian-Daniel Iordache received the M.Sc. degree from the Faculty of Electrical Engineering, Politehnica University of Bucharest, Bucharest, Romania, in 2006, and the Ph.D. degree in electrical and computer engineering from Instituto Superior Tecnico, Lisbon, Portugal, in 2011.

His research activity started in 2006 at the Electrical Engineering Research Center, Bucharest, where he worked on several national and European projects dedicated to microelectromechanical systems and high-frequency circuits. From 2008 to 2011, he was a Marie Curie Fellow with the Hyperspectral Imaging Network project funded by the European Commission under the Sixth Framework Programme. Since 2010, he is a member of the Hyperspectral Computing Laboratory research group, University of Extremadura, Cáceres, Spain. For his research activity and social implication, he was awarded with the prize The Romanian Student of the Year in Europe 2011, offered by the League of Romanian Students Abroad. He is currently carrying out his research as a postdoc researcher at the Flemish Institute for Technological Research, Center for Remote Sensing and Earth Observation Processes (TAP), Mol, Belgium. His research is focused on hyperspectral unmixing, with emphasis on the sparse characteristics of the solutions.

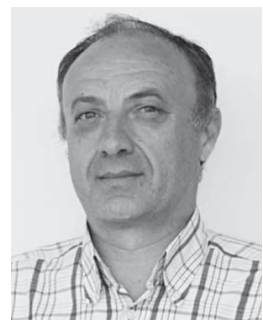

José M. Bioucas-Dias (S'87-M'95) received the E.E., M.Sc., Ph.D., and Agregado degrees in electrical and computer engineering from Instituto Superior Técnico (IST), the engineering school of the Technical University of Lisbon, Lisbon, Portugal, in 1985, 1991, 1995, and 2007, respectively.

Since 1985, he has been with the Department of Electrical and Computer Engineering, IST. He is also a Senior Researcher with the Pattern and Image Analysis group at the Telecommunications Institute, which is a private nonprofit research institution. His research interests include inverse problems, signal and image processing, pattern recognition, optimization, and remote sensing. He was and is involved in several national and international research projects and networks. He has authored and co-authored many journal and conference papers.

Dr. Bioucas-Dias is an Associate Editor of the IEEE TRANSACTIONS ON Image Processing, an Associate Editor of the IEEE TRAnsactions on CIRCUITS AND SYSTEMS, and a Guest Editor of two IEEE special issues (IEEE TGRS and IEEE JSTARS). He was the General Co-Chair of the 3rd IEEE Workshop on Hyperspectral Image and Signal Processing: Evolution in Remote Sensing-Whispers'2011 and has been a member of program/technical committees of several international conferences. 


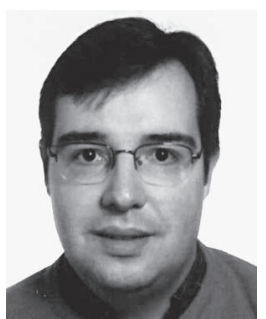

Antonio Plaza (M'05-SM'07) received the M.S. and $\mathrm{Ph} . \mathrm{D}$. degrees in computer engineering from the University of Extremadura, Caceres, Spain.

$\mathrm{He}$ was a Visiting Researcher with the Remote Sensing Signal and Image Processing Laboratory, University of Maryland Baltimore County, Baltimore, with the Applied Information Sciences Branch, Goddard Space Flight Center, Greenbelt, MD, and with the AVIRIS Data Facility, Jet Propulsion Laboratory, Pasadena, CA. He is currently an Associate Professor with the Department of Technology of Computers and Communications, University of Extremadura, where he is the Head of the Hyperspectral Computing Laboratory. He was the Coordinator of the Hyperspectral Imaging Network, a European project designed to build an interdisciplinary research community focused on hyperspectral imaging activities. He has been a Proposal Reviewer with the European Commission, the European Space Agency, and the Spanish Government. He is the author or coauthor of around 350 publications on remotely sensed hyperspectral imaging, including more than 70 journal citation report papers, 20 book chapters, and over 200 conference proceeding papers. His research interests include remotely sensed hyperspectral imaging, pattern recognition, signal and image processing, and efficient implementation of large-scale scientific problems on parallel and distributed computer architectures.

Dr. Plaza has coedited a book on high-performance computing in remote sensing and guest edited seven special issues on remotely sensed hyperspectral imaging for different journals, including the IEEE TRANSACTIONS ON GeosienCE AND REMOte SENSING (for which he serves as Associate Editor on hyperspectral image analysis and signal processing since 2007), the IEEE Journal of SElected TOPICS IN APPLIEd Earth ObSERVATIONS AND REMOTE SENSING, the International Journal of High-Performance Computing Applications, and the Journal of Real-Time Image Processing. He has served as a reviewer for more than 280 manuscripts submitted to more than 50 different journals, including more than 140 manuscripts reviewed for the IEEe Transactions on Geoscience and Remote Sensing. He has served as a Chair for the IEEE Workshop on Hyperspectral Image and Signal Processing: Evolution in Remote Sensing in 2011. He has also been serving as a Chair for the SPIE Conference on Satellite Data Compression, Communications, and Processing since 2009, and for the SPIE Remote Sensing Europe Conference on High-Performance Computing in Remote Sensing since 2011. He is a recipient of the recognition of Best Reviewers of the IEEE GEOSCIENCE AND REMOTE SENSING LETTERS in 2009 and a recipient of the recognition of Best Reviewers of the IEEE TRANSACTIONS ON GEOSIENCE AND REMOTE SENSING in 2010. He is currently serving as Editor of the IEEE Transactions on Geoscience and Remote Sensing. 